\section{Streiflichter Urbaner Diakonie}

Christoph Sigrist

Vom 14.-16. Juni 2002 diskutierten Fachexpertinnen und -Experten in Heidelberg darüber, wie der diakonische Auftrag der Kirchen, der diakonischen Werke und Gruppen in Zukunft angesichts der Transformationsprozesse in der Gesellschaft mit Fokus auf die Städte wahrzunehmen ist. Diakonie in der Stadt war das Thema. ${ }^{1}$ Diese Tagung kann als Initialzündung für die Entwicklung des Forschungsgebietes Urbane Diakonie gesehen werden, das in der Dozentur für Diakoniewissenschaft der Universität Bern seit Jahren einen Schwerpunkt darstellt und durch meine dipolare Anstellung in Praxis und Theorie als Pfarrer am Grossmünster sowie als Dozent für Diakoniewissenschaft ihre innere Struktur der Kongruierung von Theorie und Praxis sowie der Interdisziplinarität nach aussen spiegelt. Die Citykirchenarbeit in Deutschland und der Schweiz, zusammen mit Holland, beschäftigte sich im letzten Jahrzehnt vermehrt mit der Stadt. 2014 wurde das Projekt Urbane Diakonie dann endgültig auf den Schild der intensiven Forschungsarbeit gehoben. Auf der Webseite der Projektarbeit in Zürich wird der Begriff Urbane Diakonie kurz vorgestellt:

Die Urbane Diakonie handelt nach dem Motto «Gemeinsam füreinander im Quartie»» und spannt ein Netz für Beratung, Betreuung, Unterstützung und Hilfestellung

1 Heinz Schmidt / Renate Zitt (Hg.), Diakonie in der Stadt. Reflexionen - Modelle Konkretionen, Stuttgart 2003.

2 Vgl. Friedrich Brandi-Hinrichs / Wolfgang Grünberg ( $\mathrm{Hg}$ ), stadt-plan. Sichtweisen auf Hamburg, Kirche in der Stadt Bd. 12, Hamburg 2005; Christoph Sigrist u.a (Hg.), Stadtliturgien. Visionen, Räume, Nachklänge, Kirchen in der Stadt Bd. 22, Berlin 2016

Jahrbuch Diakonie Schweiz 1 (2017) - ISSN 2504-3994

Dieser Text ist lizenziert unter einer Creative Commons Namensnennung 4.0 International Lizenz (CC BY 4.0): (https://creativecommons.org/licenses/by/4.0/). für Angehörige von Gesellschaftsgruppen mit besonderen Anliegen. Sie stellt das solidarische Handeln in den Mittelpunkt ihrer Tätigkeit und verbindet junge und alte Menschen im städtischen Quartier. ${ }^{3}$

In diesem programmatischen Auftritt werden die beiden Brennpunkte der Urbanen Diakonie festgehalten: Es geht einerseits um das helfende Handeln kirchlicher und diakonischer Werke im Gesellschaftsraum der Stadt, also helfendes Handeln, das aus dem jüdisch-christlichen Traditionskreis persönlich motiviert, institutionell begründet oder unternehmensgeschichtlich prägend seine Wirkung zeigt. ${ }^{4}$ Anderseits wird dieses helfende Handeln in seiner konstitutiven Kontextualität ${ }^{5}$ mit besonderem Bezug auf die städtischen Transformationsprozesse in den Blick genommen, die in der Stadtsoziologie vor allem mit dem zunächst diffusen Begriff der «Urbanität» umschrieben werden: Die Wiederentdeckung des Städtischen hat auch die Diakonie erfasst. Ein interdisziplinäres Forschungsteam der ETH Zürich hat kürzlich mit Blick auf die Metropolitanregion Zürich «Urbane Qualitäten» herausgearbeitet und dabei «Urbanität» wie folgt definiert:

«In einem engen Sinne beschreibt Urbanisierung den Prozess der Produktion der gebauten Umwelt, also den Bau von Häusern, Produktionsanlagen und Infrastruktureinrichtungen mit ihren gesellschaftlichen Implikationen. In einem weiteren Sinne lässt sich Urbanisierung als eine umfassende Transformation der Gesellschaft verste-

3 http://www.urbanediakonie.ch/CMS/de-CH/Allgemein/Urbane-Diakonie.aspx (letzter Zugriff am 4.1.2017)

4 Vgl. zum hier verwendeten Diakoniebegriff: Heinz Rüegger / Christoph Sigrist, Diakonie - eine Einführung. Zur theologischen Begründung helfenden Handelns, Zürich 2011.

5 Vgl. zur Kontextualität der Diakonie, die konstitutiv für das helfende Handeln ist: Christoph Sigrist, Das Eigene in der Diakonie: Matthias Mettner / Joseph Jung, (Hg.), Das eigene Leben - jemand sein dürfen, statt etwas sein müssen, Zürich 2015 , $45-58$. 
hen, die eng mit der Industrialisierung verbunden ist. Dabei geht es nicht nur um die industrielle Produktion von Gütern, um Fabriken und Logistikanlagen, sondern umfassend um die industrielle Organisation der Gesellschaft.» ${ }^{6}$

Urbane Diakonie als helfendes Handeln in der Stadt ist diesen Transformationsprozessen in grundlegender Weise unterworfen, ohne diese Prozesse zu bedauern oder zu loben. Diese Prozesse laufen ab, ermöglichen Chancen, bergen Risiken und verursachen neue Herausforderungen im Bereich von Exklusion und Inklusion. Das heisst konkret: Urbane Diakonie sucht einerseits in diesen Prozessen in Quartieren gemeinsam und füreinander Wege zu einer neu ausgestalteten Solidarkultur, einer Kultur der Sorge miteinander und füreinander mit besonderem Blick auf diejenigen Menschen, Gruppen und Schlüsselpersonen, die bei diesen Exklusions- und Inklusionsprozessen von der Teilhabe am gesellschaftlichen Leben ausgeschlossen werden oder schon lange sind. Anderseits bringen Menschen mit ihrem helfenden Handeln als Diakonie in diesen Prozess der Solidarität drei Ressourcen ein, die konstitutiv für diakonisches Handeln sind:

- Räume an besten Lagen in Quartieren gehören vielfach Kirchgemeinden und Pfarreien.

- Das Freiwilligen-Netz, das sich in unterschiedlichen Generationen und Herausforderungen engagiert, ist nach wie vor äusserst stark und dicht.

- Das Menschenbild ist aus der jüdisch-christlichen Tradition gewonnen und in den vier Handlungsfeldern von Kirchesein in der Stadt verankert. Es zeigt sich in Liturgie, Diakonie, Bildung und Leitung von gemeinschaftsbildendem Leben im Quartier. Es ist deshalb stark, weil es den Menschen nicht als Kunden oder als

6 Simon Kretz / Lukas Kueng (Hg.), Urbane Qualitäten. Ein Handbuch am Beispiel der Metropolitanregion Zürich, Zürich 2016, 16.
Klientel festschreibt, sondern ihn als den versteht, der er ist: ein

Bild Gottes, ermächtigt zu Liebe und Solidarität.

Im Zusammenhang mit der Erforschung dieser so verstandenen Urbanen Diakonie unternahm ich im letzten Jahr eine Forschungsreise in unterschiedliche urbane Kontexte. Die Forschungsfrage lautete: Wie zeigt sich Urbane Diakonie in unterschiedlichen städtischen Kontexten, insbesondere in Kontexten, die durch Rassismus und Apartheid eine lange exkludierende Tradition aufweisen? Denn die Arbeitshypothese lautete, dass Urbane Diakonie vor allem Teilhabeprozesse an gesellschaftlichen Transformationen in Quartieren für unterschiedliche Menschen und Gruppen einzurichten hat. Im Folgenden sollen ausgewählte Stationen in der für die Diakonie üblichen Struktur kurz vorgestellt werden: Ein Praxisbericht führt zur diakonischen Reflexion. ${ }^{7}$

\section{Streiflicht Chicago, 6.-12. Juni 2016 - therapeutisch- soziale und theologisch-liturgische Wirkkraft Urbaner Dia- konie}

\section{Therapeutisch-soziale Wirkekraft}

Margaret Nykaza gründete im Jahr 2000 das sozial-diakonische Werk Harmony, Hope and Healing. Mit Musik und der biblischen Tradition als Ressourcen werden kreative und schöpferische Räume geschaffen für Einzelne und Familien, um Resilienz und Heilungskräfte zu stärken. ${ }^{8}$

7 Entstanden sind diese Streiflichter vor Ort des Geschehens. Deshalb sind die Texte durch diese Unmittelbarkeit des Erlebens gewoben, die jedoch auch eine gewisse Tiefenschärfe zugänglich macht, die durch Betroffenheit und Berührtheit entstehen kann.

8 http://www.harmonyhopeandhealing.org (letzter Zugriff am 4.1.2017). 
Das Werk arbeitet unter anderem mit jungen schwarzen Männern zusammen, die den Übergang von der Gefangenschaft in die Gesellschaft bewältigen müssen. Daneben richtet sich das Engagement auch an schwarze, ältere Frauen. ${ }^{9}$

\section{Erfahrungsbericht}

Wir gingen in ein zerstörtes Gebäude einer ehemaligen katholischen Kirche, das nun sozial-diakonisch umgenutzt als kirchliches Zentrum des Quartiers dient und in dem ein Secondhandshop eingerichtet ist. Die Leiterin des Projekts begrüsste uns herzlich. An der Hammond-Orgel sass Davis, ein jugendlicher ehemaliger Gefangene. Die Leiterin des Theaters übte mit den mehrheitlich schwarzen Frauen ein Stück ein. Um 14.30 Uhr kam Mach, die Direktorin.

Im Theaterstück erhielten viele der Frauen eine kleine Rolle. Thematisch drehte sich alles um die Frage: Wer kommt mit dem Schiff in den Himmel und wer wird vom Teufel verführt? Den roten Faden des Stückes bildeten Gospels, die von den Frauen a capella angestimmt und von Davis mit den Akkorden unterstützt wurden. Zum Probenbeginn betete eine alte Frau. Das Gebet war eingebettet in den Zusammenhang von Begrüssung, Organisation und Einführung in das Stück. Am Schluss beendete eine andere Frau mit einem freien Gebet ebenso das Stück.

In der Pause wurde der Geburtstag von Mach, der Direktorin, gefeiert. Es wurden Hotdog und Torte verteilt. Daran nahmen auch ca. 10 Mädchen teil, die in einem Kurs von der katholischen Kirche zu peacemaker/Schlichterinnen im Quartier ausgebildet werden. Sie hatten eine weite Anreise zu bewältigen.

-

9 Die Mission, die von dem Werk und ihrer Leiterin ausgeht, lautet: «Harmony, Hope and Healing creates a safe environment where vulnerable individuals and families heal and rebuild through the restorative power of music.» http://www.harmonyhope andhealing.org/mission-and-history-2 (letzter Zugriff am 4.1.2017).
Nach der Pause folgte der zweite Probenteil. Zu einem Musikstück tanzten die alten Frauen als Gruppe und legten eine Performance hin, die ich so in der Schweiz nicht erlebt habe. Der Körper wurde zum Rhythmus. Die Schwere des Alters wurde leicht. Die Falten und Furchen der Gesichter, die ein Leben in Unterdrückung und Gewalt schildern, wurden durchlässig für den Glanz des Lachens und des Tanzens.

\section{Reflexion}

Im Bereich der Sozial-Diakonie wurden verschiedene Themen behandelt. Die Würde des alten Menschen wurde kontextuell und situativ durch die Arbeit an der Resilienz gestärkt und zum Tragen gebracht. Der Aufbau von eigenen Kräften in Körper, Seele und Geist geriet in den Fokus der Diakonie. Der Kampf in der Unterdrückung ermöglichte erst die Anhäufung von Resilienz im Verlauf des Lebens und wurde so zur Grundierung des Selbstvertrauens. Das deutet sich im Beispiel einer alten Frau an, die mir ein Foto an der Wand zeigte, auf dem sie Barak Obama gegrüsst hatte. Nachdem sie mir die Hand gegeben hatte, sagte sie: «Nun darf ich mich nicht mehr duschen [...].»

Sakralität und Säkularität verschmolzen zu einem neuen Raum: Segensraum - Seelenraum. Die Unterbrechung des Alltags schien in einem neuen Licht auf. Diese Verschmelzung war so stark, dass man von einer säkularen Sakralität sprechen kann. Das Foto mit Barack Obama und das Schiff mit Jesus wurden zu einem einzigen Bild, das den Kampf ums Überleben als Befreiungskampf malt und den Unterdrückten Brücken nachzeichnete, auf denen die Überschreitung innerlich wie auch äusserlich möglich wurde.

Von einer sakralen Säkularität kann insofern gesprochen werden, als dass der Alltag von der spirituellen Perspektive anders gedeutet wurde, nämlich als eine von Gott geheiligte Säkularität des befreiten Sklaven. Darin drückt sich die Absage an eine - von Luther so nie formulierte Zwei-Reiche-Lehre aus, die die Trennung zwischen Kirchenraum und Gesellschaftsraum vorangetrieben hat. Sie führt zur Einsicht der reformierten Reformation, dass die theologische Verheissung der Freiheit und 
der politische Kampf um diese Freiheit zwei unterschiedliche Seiten derselben Medaille sind.

Dieses Ineinanderfliessen von Sakralität und Säkularität zeigte sich in folgenden räumlichen und ausagierenden Elementen:

- Der Kirchenraum wurde als Seconhandshop umgenutzt und der Altarteil wurde zum Kirchenschiff umfunktionalisiert.

- Der Gospel wurde zum Ausdruck dieses Ineinanderfliessens. (Der Gospel gehört zum Alltag bei den Frauen. Es ist ihr normaler Ausdruck von Sinnhaftem und der Sehnsucht nach einem besseren Leben. Der Gospel ist jedoch getränkt von biblischen Bildern.)

- Die Sprache des Gebets war zudem die Sprache der Einführung in das Theater.

- Der Tanz nach dem säkularen Song wurde zum Sakraltanz der von Alter und Schwäche befreiten Frauen.

Der Gesang spielte eine besondere Rolle. Wie auch der Tanz war er verwoben mit der Existenz der Schwarzen. Tanz und Gesang bildeten den roten Faden ihres Kampfes aus Unterdrückung und Armut. Das Projekt Harmony, Hope and Healing baut auf dieser Einsicht auf: Wer singt, lebt. Wer nicht mehr singen kann, ist tot. Davon erzählt auch der Psalm 115,17: Die Toten können den Herrn nicht loben. Der Gospel ist die Sprache ihrer Seele. Wenn die Frauen sangen, dann redete ihre Seele von der Sehnsucht nach dem Himmel auf Erden. Der Gesang war der Kontext des Textes: Ich bin würdig, Gottes Geschöpf zu sein. Alle Menschen sind gleich vor Gott. Der Mensch ist befreit, gerecht und versöhnt.

In diese Sprache wurde man mündlich eingeführt: Ein 6-jähriges Mädchen sang einen Gospel alleine, mit dem Rhythmus im Körper, und die Frauen antworteten. Darin zeigte sich, dass der Gesang dialogisch strukturiert ist und auf Antwort hin ausgelegt ist. Sozial-diakonische Arbeit muss hier als Arbeit am Gesang und mit dem Gospel als ihrem spirituellen Kapital hin ausgelegt werden: Weil der Rhythmus im Gospel so eine grosse Rolle spielt, ist die Schwester des Gesangs der Tanz. Glauben wurde tanzend und singend erlebt. Tanz und Gesang sowie der
Atem Gottes sind die drei Dimensionen des Hauses, das augenblicklich überall gebaut werden kann Im Unterschied zu den in der Schweiz adaptierten Sakralpopgesängen war der Gospel hier nicht Mittel zum Zweck, Show für die Predigt, sondern Predigt in sich, Sein aus sich, Existenz im Glauben pur.

Hier konnte die Vernetzung von beiden Projekten beobachtet werden, indem ein ehemaliger Gefangener bei den alten Frauen spielte. Das ist wohl eine typische Konturierung diakonischer Arbeit und spielt unabhängig des kulturellen Hintergrundes dieselbe entscheidende Rolle.

\section{Theologisch-liturgische Wirkkeraft}

Pfr. Dr. Otis Moss ist leitender Pfarrer der Trinity United Church of Christ. ${ }^{10}$ In dieser afroamerikanischen Gemeinde hatte Martin Luther King gewirkt, und ebenfalls Barack Obama als Jugendarbeiter, als er an der Universität studierte.

Erfahrungsbericht

Am Morgen des 12. Juni 2016 besuchte ich den Gottesdienst der Trinity United Church of Christ. Beim Empfang wurde ich bereits von unterschiedlichen Freiwilligen freundlich empfangen. Der Saal war zu dem Zeitpunkt schon zu gut einem Drittel besetzt. Neben mich setzte sich Cay Evans, ehemaliger Pfarrer und Mitstreiter von Marin Luther King. Vor mir sass die Frau von Otis Moss. Der Kinderchor sang zu Beginn zwei Gospels. Die Konfirmanden setzten sich mit den Eltern im vorderen Teil ab. Otis traf erst ca. 10 Minuten nach Beginn des Gottesdienstes um 11.00 Uhr ein.

Der Gottesdienst hatte eine strenge Liturgie. Die Liturgie erfolgte jedoch in ungewohnter Weise in der umgekehrten Reihenfolge: Zuerst ein

10 https://trinitychicago.org (letzter Zugriff am 19.3.2017). 
freies Gebet, dann das Unservater, dann die Begrüssung der verschiedenen Gäste. Nachdem ich im Namen der reformierten Kirche die Grüsse übermittelt und auch von unserem Engagement gegenüber Flüchtlingen erzählt hatte, folgte die Konfirmation, dann die Sammlung der Kollekte, dann das Altargebet für all jene, die es schwer haben und gesegnet sein wollen, dann die Predigt, dann die Segnung der in der Predigt erwähnten, dann die Zusammenfassung der Predigt mit dem Segen zum Schluss. Die Dauer der Feier betrug etwa 2 1/2 Stunden, die Predigt selber dauerte 40 Minuten.

Die Liturgie wurde durch die Musik der Band und des Chores miteinander verknüpft. Eine bedeutende liturgische Präsenz hatten zudem die Freiwilligen, die beteten, sangen, Kollekten einsammelten, Plätze zuwiesen, organisierten und eine Kultur der Achtsamkeit installierten. Otis Moss war als Pfarrer präsent hinten auf dem Sitz und vorne. Die weiteren Pfarrer wie auch die Ältesten sassen vorne und engagierten sich immer wieder am Geschehen. Die Gemeinde trug Otis Moss und den Gottesdienst: Die Gemeinde war Subjekt der Liturgie in ihrer responsiven Struktur. Sie begleitete unterstützend Songs, Gospels, Predigt und Gebet. Die Verschmelzung von Säkularität und Sakralität erwies sich auch in der Gebetstruktur, die im freien Gebet eine andere Art des Gospelklanges zeigte und so die Mehrdimensionalität der Verkündigungsstruktur aufzeigte. Das Liturgieblatt bildete zugleich Information und Mission, Diakonie und Liturgie, Verkündigung und Organisation ab.

Das Aufstehen und Absitzen der Gemeinde, das Klatschen und Jubeln, das eine Kultur der Wachheit und Präsenz ermöglichte, lässt die $2 \frac{1}{2}$ Stunden kürzer erscheinen als manch einen Gottesdienst in Zürich, der nicht über eine Stunde lang dauert. Die Lieder waren einzigartig. Bis auf ein Lied waren alle Songs frei vorgetragen. Die Gemeinde sang stehend.

Der Einstieg war fliessend und von der Musik getragen. Es wurde jedoch ein Einzug in verschiedenen Staffeln vollzogen. Am Schluss war die Kirche voll, zuvorderst waren die besonderen Plätze für die Gäste.
Die Begrüssung von Cay Evans war von Otis sehr persönlich und emotional, eine rührende Geste der Dankbarkeit in Verbindung mit der Geschichte: «Sie würden nicht hier stehen, wenn Sie nicht mit Martin Luther King gekämpft hätten.» Anschliessend überbrachte ich meine Grussworte.

«Peace be with you / Friede mit euch.»

Die Gemeinde antwortete: «Und mit dir!»

«[... God bless your preacher and you, my sisters and brothers - in Swiss German: Bhüeti Gott.»

Auch ich wurde getragen von der Gemeinde. Otis Moss hatte sehr Freude und schloss an das wichtige Engagement zwischen Deutschland und Zürich mit rührenden Worten an. Nach dem Gottesdienst wurde ich von vielen auf meine Worte angesprochen, verbunden mit der Dankbarkeit, dass ich hier sei. Wir waren ca. 5-8 Weisse unter 1000 Schwarzen.

Ca. 20 junge Erwachsene wurden gleich im Anschluss an die Begrüssung nach vorne gerufen, ebenso ihre Eltern. Dann beteten die Katecheten und auch die Pfarrer zum ersten Mal. Dann gingen die Eltern wieder zu den Plätzen und Otis Moss fragte die Jugendlichen an den Katechismus anlehnend Texte aus der Bibel (10 Gebote, Seligpreisungen). Das Glaubensbekenntnis wurde dann miteinander gesprochen, dann noch wichtige Daten aus der Geschichte und Symbole der Gemeinde. Dann wurden die Eltern zum zweiten Mal nach vorne gerufen. Die Kinder mussten ein Bekenntnis ablegen, die Eltern auch, dann die Gemeinde. Zum Schluss bekamen die Einzelnen den Segen. Sehr viel Anrührendes wurde mit Applaus kommentiert.

Für zwei Kollekten warb Otis Moss sehr emotional. Es kamen ca. $4000 \$$ zusammen. Die Freiwilligen sammelten das Geld in Kübeln, zum Teil versteckten die Mitglieder ihr Geld in Briefcouverts. Das Geld wurde durch die enge Verbindung mit dem diakonischen Engagement aufgeladen und mit dem Song zu einer neuen Währung. Die Wichtigkeit des Gebets wurde in den liturgischen Rahmen integriert, so dass Geld, Geist und Gemeinschaft ineinanderfliessen. 
Vor der Predigt wurden unterschiedliche Leute mit ihren Nöten und Sorgen durch einen Diakon angesprochen. Mit Namen wurden diejenigen aufgerufen, die im Krankenhaus lagen oder die zügelten. Dann kamen weitere nach vorne und alles mündete in ein Fürbittegebet. Hier geschah wohl am intensivsten die Verbindung vom liturgischen und diakonischen Geschehen und die Fürbitte wurde zum Fenster nach draussen.

Die Predigt war der Höhepunkt und auch der Schlusspunkt des Gottesdienstes. Es wurde noch einmal der Predigttext wiederholt: Apg 20,18. Otis Moss las ihn noch einmal. Dann begann er mit der Auslegung, indem er zu Beginn immer wieder den Satz wiederholte:

«The church is not perfect, perfect, she is imperfectly.» Die Kirche ist nicht vollkommen, vollkommen, sie ist unvollkommen. Was heisst imperfekte Kirche? Es gibt schlechte Lehrer und Pfarrer, und doch geht ihr in die Schule und in den Unterricht.

Otis Moss imitierte das lange Reden des Paulus. Er begann zum ersten Mal in einen Rap zu fallen und forderte die Gemeinde auf, zu antworten. Das Bild des Jungen im Fenster wurde zum Mittelpunkt:

Was ist das für eine Gemeinde, welche die Jungen, die die Zukunft der Kirche sind, nicht in ihre Mitte zieht, sondern auf den Fensterplatz setzt? Paulus Wirken hat zur Folge, dass ein Glaubender stirbt. Was für ein «Erfolg»!? Paulus geht nach dem Wunder wieder in die Kirche und predigt weiter. Die Leute folgen ihm in die Kirche. Wer in die Kirche kommt, fällt zuerst ausserhalb der Kirche und kommt durch das Wunder der Auferstehung wieder in die Kirche. So ereignet sich Kirche: Gottes Gnade und Barmherzigkeit erweckt vom Tod und bewirkt in der Seele die Kraft, selber barmherzig und gnädig zu sein. Wer ist aus dem Fenster gefallen?

Otis Moss forderte unter Applaus die Menschen auf, nach vorne zu kommen. Seine Ehefrau spielte eine Schlüsselrolle: Sie nahm zwei Frauen an der Hand. Hier begann die Band zu spielen, immer wieder denselben Ton. Otis Moss beschloss die Predigt mit einem Segensgebet und der trinitarischen Formel und dem Amen.
Aus homiletischer Perspektive sind besonders interessante Beobachtungen festzuhalten:

- Der Einbezug der eigenen Geschichte: Die Kirche hatte beim Widerstand auch eigene Menschen sterben lassen. Der Blick richtete sich zuerst zur eigenen Geschichte: Der Kontext der Bibel wurde zuerst in den Text der eigenen Gemeinde gelegt.

- Die Auswahl dreier Fakten aus dem Text: Lange Predigt und Schlaf, Fall aus dem Fenster, und die Aufnahme der weiteren Predigt mit dem Brechen des Brotes.

- Otis Moss hatte mit der Hand in ein Buch seine Predigtgedanken geschrieben. Er legte das Buch auf die Kanzel in der Mitte und ging immer wieder einige Meter nach hinten. Er modulierte mit der Sprache, indem er wiederholte, ablas, frei redete und immer wieder den Kontakt mit der Gemeinde suchte.

- Er spielte mit der Tonalität, mit der Lautstärke und der Geschwindigkeit. Dreimal rappte er sich in ein Feuer mit der Aufnahme von Motiven aus dem Text, die Gemeinde nahm den Ball auf und trieb Otis Moss an mit Applaudieren, mit Ausrufen wie «that's good» und mit Jubelrufen oder untermauerndem «Jee».

- Humor und der Einbezug von spontanen Einfällen sowie improvisierenden Einschüben ergänzten die 40-minütige homiletische Performance, bei der sich Otis Moss mehrmals mit einem weissen Tuch den Schweiss von der Stirne wusch.

- Der Schluss war mit der Band abgesprochen und endete in einer segnenden Gebetshaltung eines Gospels.

- Zum Schluss kamen diejenigen, die vor der Kirche gefallen sind, nach vorne und hielten sich die Hände. In dem Augenblick fasste Otis Moss mit den Bildern des Textes noch einmal die wichtigsten Punkte fest:

Die Kirche ist nicht perfekt, die Jungen gehören in die Mitte, keiner kommt in die Kirche ohne den Fall draussen, die Präsenz Christi zeigt sich in der Auferstehung und im Geist Gotte, der lebendig macht und befähigt, zu leben und zu lieben. 
Diese beiden Begriffe schlossen den Gottesdienst ab und er hielt spontan einen Segensteil, indem er die ganze Gemeinde, die rundherum stand, segnete.

Reflexion

Die intensive Verbindung zwischen Diakonie und Liturgie bekamen durch die gottesdienstlichen Elemente ihren starken Ausdruck:

- Das kirchgemeindliche Leben wurde liturgisch durch eine von allen gelesene Fürbitte wie auch die Nennung der Namen von Menschen, die krank und in Not waren, diakonisch eingebettet (Diakonisierung der Gemeinde und Gemeindewerdung der Diakonie). ${ }^{11}$

- Die Kollektenansage und das zentrale Einziehen mitten im Gottesdienst nahmen die Gemeinde in den liturgisch-diakonischen Dienst hinein.

- Durch die aktive Teilnahme der Menschen mit Not am Kanzeltisch wurde die Vulnerabilität und Verletzlichkeit des menschlichen Lebens sichtbar gemacht.

- Die diakonisch ausgerichtete Gestaltung des Liturgieblattes unterstrich den besonderen Charakter des Gottesdienstes.

- Die Aktivierung der Gemeinde im Gesang und auch mit dem Tanz half der Gemeinde, ihrem Engagement im Glauben Ausdruck zu verleihen.

- Die befreiungstheologischen Einwürfe bei der Vorstellung der Gäste öffneten das Geschehen für einen weiteren Horizont (Schritte zum Diakonentum aller Gläubigen).

Der Gottesdienst war durch das Call-and-Response-Prinzip des Gospels getragen und fundiert. Das bedeutet: Subjekt der Liturgie war die Ge-

11 Vgl. Jürgen Moltmann, Diakonie im Horizont des Reiches Gottes. Schritte zum Diakonentum aller Gläubigen, Neukirchen-Vluyn 1984. meinde. Subjekt der Verkündigung war die Gemeinde in dem Sinn, dass die Interaktion zwischen hörender Gemeinde und auslegendem Prediger einen Verstehensraum geschaffen hat, indem die Menschen in der Spannung von kollektivem Einverständnis und individueller Erkenntnis sich anders, neu und überraschend anders zu verstehen beginnen im Kontext des Wortes Gottes. Damit transzendiert das Call-and-Response-Prinzip zum geistgewirkten Sakralraum,

- indem Gott redet und die Gemeinde antwortet,

- indem die Gemeinde redet und Gott antwortet,

- indem ich rede und Gott antwortet und

- indem Gott redet und ich antworte.

Diese Mehrdimensionalität des Call-and-Response-Prinæips kann demnach als aus der reformierten Tradition gewonnene Inspirationslehre verstanden werden, um die Hermeneutik der Öffentlichkeit des Evangeliums hör- und sichtbar zu machen: Auslegung des Evangeliums ist öffentlicher Dienst vor Gott und der Welt von Gemeinde und Prediger zugleich. Diese Öffentlichkeit ist Grund für das Öffnen des Kirchenraumes hin zur Not in der Welt.

Durch die aktive Rolle der Gemeinde wurde eine Emotionalität über das ganze Geschehen gelegt: Gott wurde in Emotionen erfahrbar, weil er das Herz mit seiner Liebe berührte. Diese Herzensberührung war zugleich das emotionale Fenster zum Widerstand gegen die harten Herzen, die voller Schmerz und Scham verletzten, töteten und unterdrückten. Die Emotionalität war der Kontext des Intellekts mit seinem Text, so dass zwischen den Zeilen gelesen werden konnte. Mit dieser Emotionalisierung der intellektuellen Leistung des Verstehens ging die Bedeutung der einzelnen herausragenden Personen und die Einbettung ihrer Verehrung in den Gottesdienst als Glanz der Verehrung Gottes einher. Die in der reformierten Tradition eingelagerte Kritik an der Personalisierung des Gottesdienstes nimmt eine Gefahr der Heroisierung auf. Doch sie schüttet das Kind mit dem Bade aus, indem die inkarnatorische Dimension des Glaubens gekappt wird. Wie denn, wenn nicht durch einen Menschen kann die Verehrung Gottes menschlich sagbar werden?! 
Abschliessend kann der Gottesdienst von der Trinity United Church of Christ als herausragendes Beispiel dafür gelten, dass überall dort, wo Gottes Wort verkündet wird, sich Gemeinde in besonderer Weise bildet. Besonders war diese Art und Weise deshalb, weil innerhalb des Versammlungssaales ein neuer Raum entstand, der durch den Gesang, die Emotionalität und die Authentizität des Predigers, die Anwesenheit von vielen Menschen und die Wertschätzung des vulnerablen Lebens bestimmt und ausgestaltet wurde. Die Relationalität des Raumes bekam durch die Inspiration des Predigers eine weitere Dimension hinzu, die ihn zu einem Heterotop für die Seele werden liess. Der Gottesdienstraum wurde so zum Segensraum für die verletzliche und verletzbare Seele. Die Kraft Gottes wurde als seelische Kraft für den Kampf und den Schmerz der Seele so eingelegt, dass sie eine Woche - bis zum nächsten Gottesdienst - überlebte. Diese seelische Kraft Gottes wirkte individuell so, dass sie das Individuum des Glaubens ins Netzwerk der Gemeinde einband und die Lebenswelten miteinander verknüpfte.

In mehreren Gesprächen mit Otis Moss näherte ich mich an seinen befreiungstheologischen Ansatz heran. Diese Einsichten wurden durch den Besuch des Gottesdienstes vertieft. Aufgrund eines Songs von Aaron Thibeaux «T-Bone» Walker mit dem Titel «Stormy Monday» schrieb Otis Moss:

«The pain of the week is connected to the sacred service of Sunday. There is no strict line of demarcation between the existential weariness of a disenfranchised person of color and the sacred disciplines of prayer, worship, and service to humanity $[\ldots]$ America is living Stormy Monday, but the pulpit is preaching happy Sunday.» ${ }^{12}$

In dieser engen Verbindung von Schmerz im Alltag mit dem «heiligen Dienst» am Sonntag liegt nach Otis Moss einerseits die Kraft des Blues.

12 Otis Moss, Blue Note Preaching in a Post-Soul World. Finding Hope in an Age of Despair, Louisville (Kentucky) 2015, 4
Anderseits lehnt er aus dieser Perspektive die traditionellen Vorstellungen (notions) von heilig und säkular ab. Darin liegt eine der wichtigen Differenzerfahrungen zwischen der amerikanischen evangelischen Frömmigkeit und der westeuropäischen Frömmigkeit eingelagert, die es für den sozial-diakonischen Bereich nutzbar zu machen gilt. Für den liturgischen Aspekt ist die Inkulturation des Bluesgebets der Grund, dass Gottesdienst und politisches Engagement ineinanderfliessen. Das führt zu einer besonderen Legierung spiritueller Existenz im Alltag und gestaltet das liturgische Geschehen dialogisch aus (Call-and-Response-Prinzip). Rhythmisch führt es in den Tanz der Auslegung. Doch was heisst diese nicht zu eng gedachte Verbindung von Schmerz und heiligem Dienst für den diakonischen Auftrag im Kontext amerikanischer schwarzer Frömmigkeit mit dem Fokus auf relevante Einsichten für die Schweiz? Im Folgenden greife ich drei Perspektiven auf: Ekklesiologisch, homilitisch/liturgisch und kirchengeschichtlich.

Ekklesiologisch ist die Trennung von Liturgie und Diakonie nicht nur zu überwinden, sondern in die kybernetischen Prozesse bei den Kirchenreformen einzubauen. Es geht nicht an, dass Kirchgemeinden ihren diakonischen Auftrag sogenannt «auslagern» wollen und ihn an institutionelle Kräfte delegieren wollen. Der Schmerz des Alltags ist konstitutiv für die Heilung am Sonntag: Ohne Schmerz keine Heilung und ohne Heilung kein Ertragen des Schmerzes. Diese Verschränkung äussert sich in mehreren Dimensionen.

- Ökonomisch: 5\% jedes Budgets sind diakonisch auszuweisen.

- Berufspolitisch: Sozial-Diakonie und Pfarramt, Kantorat und Katechetik haben sich zu einem ekklesiologischen «think-tank gelebter und lebendiger Diakonie» zu vereinen.

- Gesamtkirchlich: Jede konzeptionelle Reflexion ohne Praxis ist keine theologisch verantwortete Arbeit an der Auslösung von diakonischen Prozess in Kirchgemeinden, diakonischen Institutionen und Werken.

Homiletisch und liturgisch werden Perspektivenwechsel sowie die Kontextualität des Glaubens zum hermeneutischen Schlüssel für das gesamte 
Geschehen. Otis Moss geht von Psalm 137 aus. ${ }^{13}$ Darin spiegelt sich das extra nos reformatorischer Einsicht, dass ich erst durch das Ausserhalb von mir das Innerhalb von mir zu verstehen lerne. Dieser Perspektivenwechel hin zum andern gehört jedoch zur Urentscheidung diakonischen Handelns und wird in Lukas 10 im jesuanischen universalen Hilfeethos grundlegend konturiert (Luk 10,36). Daraus folgt: Die Erfahrung des Schmerzes der Notleidenden gibt dem Helfenden jene Realität, so dass sie nicht über die eigene Realität sprechen müssen. Homiletisch gilt es, diese fremde Realität so zu Wort kommen zu lassen, dass der Hörende sie zwischen die Zeilen der eigenen Realität zu schreiben vermag. Der Text der Bibel wird im homiletischen Prozess zum Kontext des Textes des eigenen Lebens. Die Kontextualität der Bibel ist demnach paradox zu verstehen: Einerseits gilt es, den Text der Bibel auszulegen in den heutigen Kontext der Zeit. Anderseits wird der Bibeltext zum Kontext der Auslegung des Textes des Zuhörers. Darin spiegelt sich im westeuropäischen Kontext das empirische Ende wider, das sich mit Ernst Lange am klarsten zeigte: Der Text der Predigt ist das Leben. Aus dem Kontext der schwarzen Theologie lässt sich die komplexe Struktur der Kontextualität des homiletischen Geschehens deutlich nachzeichnen und führt zu reformatorisch-reformierten Einsichten.

Kirchengeschichtlich lassen sich mit Blick auf Huldrich Zwinglis Theologie und Existenz unterschiedliche Verbindungen aus dieser von Otis Moss auf den Punkt gebrachten Erkenntnisse nachzeichnen: Die Einführung der Almosenordnung ist ein Indiz dafür, dass für Huldrich Zwingli der Schmerz des Armen konstitutiv für die Auslegung des Evangeliums von den Armen und für die Armen war. Theologisch zeigt sich Gott in der Parteinahme für die Armen (Psalm 82). Ekklesiologisch kann die Reformation der Kirche nur als Transformation der Gesellschaft ver-

13 Moss (Anm. 12), 8: «That is Blues Speech that gives reality to my reality, though I am not to speak about my reality. This is a Blues Song.» standen werden. Ethisch hat sich die menschliche Gerechtigkeit immer an der göttlichen Gerechtigkeit zu messen. Sie ist immer demokratisch verhandelbar, kompromissgesteuert, beschränkt, begrenzt und der Situation der Zeit anzupassen. Sie ist nach humanistischen Methoden und Wissenschaften zu erkennen und einsichtig zu machen und hat immer vorläufigen, kontextuellen Charakter. Ein Grundkriterium humanistischer Einsicht ist das massvolle Verhalten mit Augenmass und auf $\mathrm{Au}-$ genhöhe. ${ }^{14}$ Es gibt keine Unterscheidung zweier Reiche, eines des Glaubens und eines der Gesellschaft, sondern sakral und säkular sind vereint. Deshalb ist es möglich,

- Kirchenräume für politische Auseinandersetzungen (Disputationen) zu öffnen,

- Kirchenräume und Kirchenkomplexe für diakonische Zwecke umzunutzen (Predigerkloster, Wasserkirche),

- aufsuchende Soziale Arbeit, liturgisches Gebet und Auslegung der Bibel zeitnah im gleichen Raum miteinander zu verbinden ${ }^{15}$ und

- Geld und Geist als zwei unterschiedliche Seite derselben Medaille zu verstehen: Geld ist immer sozial gebunden und ist gegen Wucher und Geiz zu schützen. ${ }^{16}$

14 Vgl. den Entscheid der zweiten Disputation vom 26.-28. Oktober 1523 in Bezug auf die Einführung des Abendmahles, das erst am Gründonnerstag 1525 vollzogen wurde: Huldrich Zwingli, Die Akten der zweiten Disputation vom 26.-28. Oktober 1523 Huldreich Zwinglis sämtliche Werke Band 2, Corpus Reformatorum 89, Leipzig 1908, 681ff.

15 Vgl. meine Ausführungen in KirchenDiakonieRaum zu der Typologie des Kirchenraumes in Kapitel 5: Christoph Sigrist, Kirchen Diakonie Raum. Untersuchungen zu einer diakonischen Nutzung von Kirchenräumen, Zürich 2014.

16 Vgl. den Fünfprozent-Ansatz zum Zins in der Schrift «Menschliche und göttliche Gerechtigkeit» (1523) und die Gutachten für das Zinswesen (Mai und August 1525): Huldrich Zwingli, Von göttlicher und menschlicher Gerechtigkeit: Huldreich Zwing- 
Die Einsichten der Bibel sind dem Volk zu erschliessen. Dieser Prozess des Erschliessens geschieht in der hermeneutischen Struktur des Call-andResponse-Prinzips und kann mit dem Blues nachgezeichnet werden. Die Auslegung ist auf den öffentlichen Raum kontextuell angewiesen: Der von der Gemeinde mandatierte Ausleger legt das Wort der Bibel der hörenden Gemeinde aus. In dieser Interaktion von hörender Gemeinde und auslegendem Prediger erschliesst sich die Bibel aus sich selber und wird im aktuellen Vollzug zum Wort Gottes. Die Inspiration der Bibel ist situativ, kontextuell und kontingent. Dieser pneumatologische Ansatz ist auf das Call-and-Response-Prinzip angelegt und widerspricht zentral der persönlichen Bibellektüre im stillen Kämmerlein.

Sozial-diakonisch zeigt sich, dass sich kirchliche Soziale Arbeit in einem pluralen, urbanen Kontext aus dieser engen Verbindung heraus zu entwickeln und zu bewähren hat. Die kirchliche Soziale Arbeit gewinnt dadurch Motivation, theologische Begründung und Wirkkraft. Kirchliche Soziale Arbeit ist immer politische, prophetische Arbeit, die die Strukturen von Schmerz und Gewalt aufzeigt und aufgrund der biblischen Kontexualisierung in neue Horizonte und überraschende Zusammenhänge stellt. Dazu braucht es Menschen (Gemeinden), die Vision einer menschenfreundlichen Gesellschaft, Geld und öffentliche Kommunikation. Schliesslich geht es darum, in der Kombination von Mystik und Widerstand, Mutanfall und Gelassenheit pragmatisch in kleinen Schritten politisch-prophetisch vorzugehen, indem mit Netzwerkarbeit sondergleichen

-

lis sämtliche Werke Band 2, Corpus Reformatorum 89, Leipzig 1908, 471ff. Huldrich Zwingli, Erstes Gutachten betreffend Zehnten: Huldreich Zwinglis sämtliche Werke Band 4, Corpus Reformatorum 91, Leipzig 1927, 352ff. Huldrich Zwingli, Zwinglis zweites Gutachten betreffend Zehnten usw.: Huldreich Zwinglis sämtliche Werke Band 4, Corpus Reformatorum 91, Leipzig 1927, 536ff. Glaube und Bildung gehören zusammen: Prophezeien im Kirchenraum, Arbeitsprogramme ausserhalb der Kirche für die Bekämpfung der Armut. am Netz der Menschlichkeit gewoben wird. Die Stadt in ihrer pluralen, durch Gentrifizierung und die Exklusion-Inklusion-Debatte konturierten Struktur ist Forschungslabor neuer diakonischer Aufbrüche: Der Schmerz der Stadt bildet den Kontext für den Text der Diakonie der Kirche mit ihrer heilenden Wirkung.

\section{Streiflicht London, 13. Juni - 3. Juli 2016 - Gemeinwesen orientierte, kontextuell orientierte Wirkkraft urbaner Diako- nie}

Transformationsprozesse von Urbanität schlagen in London in grossem Masse Gentrifizierungsspuren in Quartiere und Stadtteile und verändern deren Gesicht in zum Teil dramatischem Ausmass.

«London ist traditionellerweise multikulturell, ein Anziehungspunkt für verschiedene Nationalitäten, Kulturen und Religionen und damit ein Beispiel für «Superdiversität. In London leben Menschen aus etwa 179 Ländern und es werden 300 Sprachen gesprochen.» $^{17}$

Dem Zusammenleben und der Quartier- und Gemeinwesenarbeit in den einzelnen Boroughs von London kommt wegen der ethnisch sehr vielfältigen Bevölkerung eine wichtige Rolle zu. Gemeinwesenarbeit hat in London eine lange Tradition und geht zurück auf den Sozialreformer Samuel Barnett, Pionier der Gemeinwesenarbeit und Gründer der Toynbee Hall, dem ersten Nachbarschafts- und Bildungszentrum in London im späten 19. Jahrhundert. Grundgedanke dieser Einrichtungen war, dass

17 Arbeitspapier: Zum Delegationsprogramm «Zürich meets London»: Hintergrundinformationen zu London, Organisation: Stadtentwicklung (Aussenbeziehungen und Wirtschaftsförderung), Stadt Zürich, und Amt für Wirtschaft und Arbeit des Kantons Zürich, 17.-20. Mai 2016, 11-12, zu beziehen bei: www.christophsigrist.ch. 
die Ursachen von Armut und sozialer Ungerechtigkeit nur gemeinsam mit den Betroffenen bekämpft werden können. Es war das Ziel, Probleme vor Ort (z.B. mangelnde Integration oder Chancenungerechtigkeit, Arbeitslosigkeit, Armut) aufzugreifen, langfristig zu lösen und dadurch die Lebensqualität dieser Menschen zu verbessern. Dabei ging es immer auch um eine Mobilisierung der Menschen zur Eigeninitiative, um Unterstützung der schon vorhandenen lokalen Gruppen und um Vernetzung der verschiedenen Institutionen, Initiativen und Einzelpersonen.

Mit Blick auf die Quartiersentwicklung sowie deren Transformationsprozesse ist Southwark Forschungslabor für die Fragestellung der gemeinwesenorientierten Urbanen Diakonie, insbesondere die Arbeit von Bede House. ${ }^{18}$

Erfahrungsbericht

Der Zweck des Aufenthalts bestand darin, vor Ort einen Einblick zu erhalten über verschiedene erfolgreiche Ansätze und Formen der Integration und der Quartier- und Gemeinwesenarbeit in einer sehr heterogenen europäischen Stadtgesellschaft. Konkret besuchte ich das Community Center Bede House im Stadtteil Southwark.

Southwark hat eine ethnisch vielfältige und junge Bevölkerung. 2012 waren von 293'530 Einwohnerinnen und Einwohnern ca. 58 Prozent unter 35 Jahren. Southwark hat den höchsten Anteil an Bewohnerinnen und Bewohnern, die in Afrika geboren sind (12.9\%), und einen signifikanten Bevölkerungsanteil aus Lateinamerika. 75 Prozent der schulfähigen Kinder sind dunkelhäutig oder stammen von ethnischen Minderheiten ab. In Southwark werden mehr als 120 Sprachen gesprochen. In elf Prozent der Haushalte gibt es keine Person, die Englisch als Muttersprache hat. Southwark hat die neunthöchste Bevölkerungsdichte in England und Wales mit 9'988 Einwohner/innen pro Quadratkilometer.

18 www.bedehouse.org (letzter Zugriff am 4.1.2017).
Bede House ist eine Institution, die sich seit 1938 vor Ort für wohltätige Zwecke einsetzt und Menschen unterstützt, um ihnen und ihren Familien ein besseres Leben zu ermöglichen. ${ }^{19}$ Als lokal tätige Organisation kann Bede House rasch auf sich verändernde Bedürfnisse im Einzugsgebiet reagieren. Bede House konzentriert sich hauptsächlich auf vier Dienstleistungen:

- Hilfe für Erwachsene mit Lernbehinderungen: gemeinsame Treffen mit sozialen, sportlichen und künstlerischen Aktivitäten, Angebote für individuelle Unterstützung, spezielles Trainingsprogramm über 12 Wochen täglich inkl. Arbeitserfahrungen, Unterstützung bei der Arbeitssuche, Freiwilligenarbeit, Arbeit im Bede Café;

- Arbeit mit Jugendlichen im Alter von 8 bis 19 Jahren: Aktivitäten, die Spass machen und herausfordern (im 4-WochenRhythmus: Planen, eine Fähigkeit trainieren, ein bedeutsames Thema diskutieren, Filmnacht), Musikstudio und BikeWerkstatt, Tagesausflüge, kurze Aktivferien in England oder Wales;

- Projekt Starfish für Opfer von häuslicher Gewalt oder Missbrauch: Unterstützung bei der Gewährleistung von Sicherheit, rechtliche Informationen, Vertrauensaufbau, falls nötig Einbezug der Polizei;

- örtliches Engagement mit dem Projekt «Big Local South Bermondsey Partnership».

Gemäss offizieller Statistiken gehören einige von Southwarks Haushalten zu den 10 Prozent der Bedürftigsten in London. Bede House bemüht sich, so vielen Menschen wie möglich die nötige Unterstützung zu gewähren, indem sie Eigenverantwortlichkeit und Unternehmertum fördert.

\footnotetext{
19 http://www.bedehouse.org.uk/ (letzter Zugriff am 4.1.2017).
} 
Reflexion

Die Theorie der 4 Räume des Helfens ist auch im Kontext von London plausibel. ${ }^{20}$ Bede House kommt von der Settlement-Bewegung aus den 60er Jahren.

Deren Grundsatz lautete: Wir gehen in das Quartier, um dort zu wohnen (settle). Wi wollen mit einem geteilten Leben einen neuen Ansatz in der sozialen Arbeit umset zen; es ist ein Geben und ein Nehmen miteinander. Dieser Grundsatz stimmt durch all die Transformationen einer Gesellschaft und entspricht auch dem christlichen Ansatzes des Hauses.

Zu Beginn war es nur ein Haus und die Initiative einer Kirchgemeinde und mehrerer Pfarrer. Nun ist es säkularisiert und die Arbeit wird nich mehr theologisch, sondern philosophisch weltanschaulich begründet. Bis 2002 war das Quartier bestimmt von der Spannung zwischen Schwarzen und Weissen, jetzt sind es eher die Spannungsfelder arm/reich und jung/alt.

Durch den Bau der U-Bahn-Linie entstand im Gentrifizierungsprozess eine kosmopolitische Gesellschaft mit pluralem Hintergrund. Themen, die nun im Quartier anstehen sind Jugendarbeit, Arbeit mit Behinderten, häusliche Gewalt und Gemeinschaftsleben. In dem Projekt Lear ning Disabilities werden Behinderte ermächtigt, bei älteren Menschen Hilfeleistungen zu erbringen. Ein Beispiel der Projektarbeit zeigt den Einsatz gegen häusliche Gewalt:

Eine 30-jährige Frau wurde von ihrem Mann wie eine Sklavin gehalten. Der Mann verfolgte sie überall hin. Durch ein Telefon wurde der Mitarbeitende auf den Arzt aufmerksam, den sie - auch unter Aufsicht ihres Mannes - besuchte. Der Mitarbei tende nahm Kontakt auf. Bei einem nächsten Besuch wartete er auf dem FrauenWC. So kam es zu einem ersten Kontakt. Auf diesem Frauen-WC, ohne die Aufsicht des Mannes, konnte die Frau mit ihrer Resilienz ermächtig werden, nach 14 Tagen den Moment abzuwarten und aus dem Haus zu fliehen und in ein Frauenhaus zu ziehen.

Die Beziehung zum Staat wurde in den letzten 16 Jahren durch die Kontrakte immer komplexer: Die Kontrakt-Struktur differenzierte die soziale Arbeit durch Bürokratisierung und Administration so sehr, dass die eigentliche soziale (und diakonische) Struktur fast nicht mehr möglich wurde. Gerade das letzte Fallbeispiel zeigt deutlich die Wirkkraft Urbaner Diakonie im Zusammenspiel mit anderen Anbietern zusammen. Sie muss sich durch die Ökonomisierung und Strukturierung der Sozialen Arbeit des Staates hindurch Wege zum tatsächlich betroffenen Menschen suchen.

\section{Streiflicht Pretoria, 8.-22. August 2016 - theologisch- reflexive und liturgisch-therapeutische Wirkkraft Urbaner Diakonie}

An der Universität Pretoria lehrt Johannes Meylahn praktische Theologie. Seine Eltern sind Flüchtlingskinder aus dem Zweiten Weltkrieg, die dann in den 50er Jahren in die Mission nach Südafrika gingen. Johannes Meylahn sagt über sich:

«Ich bin weder Südafrikaner, noch Deutscher noch Engländer [...]. Ich habe gelernt, dass weder Religion noch Kultur identitätsstiftend im Sinne von Monokultur sein können. Das Christentum ist in dem Sinne verstanden eine Anti-Religion, denn in

20 Vgl. Rüegger / Sigrist (Anm. 4), $241 \mathrm{ff}$. 
Christus bin ich befreit, mich in einer Kultur oder einer Religion festzuschreiben, ich habe ein religionskritisches Moment in meiner DNA.» ${ }^{21}$

Vor 1994 hatte sich die lutherische Kirche passiv gegenüber der Apartheid verhalten. Nach 1994 wurde in den Kirchgemeinden proklamiert: Lutherisch ist deutsch. Dasselbe Phänomen ist nun auch an der Universität zu beobachten: Die Regierung hat zum ersten Mal verlauten lassen, dass Afrikaans nicht mehr offiziell an der Uni gelernt wird, obwohl in der Verfassung steht, man solle dies tun, sofern es praktisch möglich. Die Reformierten Kirchen als Begründer der Fakultät wehren sich vehement. Sie sagen: Afrikaans gehört zur reformierten Identität. Dabei haben auch die «colors», also diejenigen, die sich aus gemischten Kulturen entwickelten, Afrikaans als Muttersprache. Die Sprache wird für die nationalistischen und identitätsstiftenden Momente instrumentalisiert und lässt sich so als Instrument von Exklusion verstehen.

Diakonie als helfendes Handeln hat in diesem Kontext eine hermeneutische, sprachpsychologische Funktion, indem sie Sprache innerhalb von Sprachdiversität bildet und lernt. Die Sprachdiversität macht die Inklusion als identitätsstiftende Differenzerfahrung sprachfähig. Mit Sprache wird Welt gebildet, deshalb liegt der Diakonie der schöpfungstheologische Gedanke zugrunde, dass durch Wort Welt erschaffen wird. Die christologische Zuspitzung in Joh 1,14 hat eine religionskritische und deshalb auch eine kirchenkritische Funktion: Der Glaube an Christus wird nicht abgrenzend als Identitätsmerkmal, das mich in Abgrenzung zum anderen sieht, sondern als kritisches Potential kategorischer Festbindung von Kultur, Kirchesein und Religion an die eigene Identität verstanden.

21 Aussage von Johann-Albrecht Meylahn in einem persönlichen Gespräch. Vgl. zu seinen Publikationen: http://up-za.academia.edu/JohannAlbrechtMeylahn (letzter Zugriff am 4.1.2017)
Die Wahrheits- und Versöhnungskommission, von Tutu geleitet, hatte ihre Empfehlungen nicht umsetzen können. Die Kritik sagt, dass sie damit die breite Masse mit einem Trick hintergangen habe. Die Kritik an der Kritik sagt, dass Mandela und Tutu dies wirklich geglaubt hatten. Eine Empfehlung der Kommission lautete: Begegnung ist nötig; es ist nötig, dass man aufeinander hört. Denn dann sei Restitution gar nicht mehr nötig. Michael Lapsley hatte dies unter dem Motto healing of memories verbreitet. Diesen Auftrag haben die Kirchen in den letzten 20 Jahren nicht erfüllt. Diakonie hat jedoch ihre gemeindebildende und gesellschaftsformierende Wirkung, wenn sie solche Orte und Räume der Begegnung schaffen kann, die heilend wirken. Kirchenräume können solche Räume sein oder werden, Umnutzungen diakonischer Art haben diese heilende Wirkung zu vollziehen. Hier sind sicher in der Differenzerfahrung der Kontextualität vergleichende Aspekte von Europa und Südafrika miteinander ins Gespräch zu bringen.

Mbeki hatte die ökonomische Falle erkannt: Südafrika ist nicht ein Kind des Kalten Krieges. Nach dem Fall der Berliner Mauer und der Freilassung von Nelson Mandela im Februar 1990 musste sich Südafrika innerhalb von Monaten im Kraftfeld der Weltgemeinschaft positionieren. Mbeki hatte das amerikanische Abkommen damals nicht unterschrieben, was Suma getan hatte. Er sprach von der SüdafrikaRenaissance und wollte als Sozial-Demokrat eine eigene Identität aufbauen. Doch die Kluft zwischen reich und arm war zu gross. Der Bruder von Mbeki, Ökonom, schrieb unlängst, dass die jetzige Regierung diese Kluft etxra so weit halten möchte, damit ihr die Wähler nicht verloren gehen. Vor allem die Bildung war in den letzten 20 Jahren ein Instrument gewesen, um diese Kluft auseinanderzuhalten. Das Bildungswesen ist sehr schwach. Die grossen Figuren kamen alle aus kirchlichen Schulen, die in der Apartheid massiv unter Druck gerieten. Diese sakrale Heilsökonomie mittelalterlichen westeuropäischen Anstriches ist eine der grossen Sünden in den letzten Jahren.

Durch das Abkommen mit den USA gingen alle Hühnerfarmen der Einheimischen zugrunde, sowie auch das Pharmawesen. Diese neue öko- 
nomische Seite des Post- oder Neo-Colonialism drängt auf die Bildung einer neuen Ökonomie. Diakonie als Instrument einer Gaben-Ökonomie (Benedict) kann hier hilfreich sein.

Transformation ist politisch zu verstehen. Südafrika ist in einem starken Transformationsprozess, deren Indiz die letzten Wahlen gewesen sind. Indikatoren sind die Gentrifizierungsprozesse in den Städten sowie in den Schulen. An der Universität sind zum ersten Mal schwarze Studierende der zweiten Generation, die den Abschluss suchen. Die Reformierte Kirche hat ein grosses diakonisches Werk, wie in Deutschland, doch in diesem Werk sind keine Pfarrer angestellt, sondern Sozialarbeiter. Wie sind solche Werke in der Transformation der Gesellschaft zu positionieren? Kirchgemeinden verändern sich von weiss zu schwarz. Wie haben sie ihre Identität zu gestalten? Dabei sind immer wieder die beiden grundlegenden Fragen in den Blick zu nehmen: Wie kann ein Leben miteinander gelingen? Wie kann Identität von Person, Gemeinschaft und Gesellschaft angesichts der Transformationsprozesse neu aufgebaut werden, indem Differenz und Diversität nicht als Gefahr, sondern als Bausteine nachhaltiger Werte verstanden werden?

\section{Sozial-diakonische Einblicke}

Das sozial-diakonische Werk Tshwane Leadership Foundation ${ }^{22}$ (TLF), gegründet von Stephan de Beer, Praktologe an der Universität in Pretoria, ${ }^{23}$ aktuell geleitet von seiner Frau, Wilma de Beer, arbeitet im Bereich verschiedener sozialer Brennpunkte in Pretoria. Gegründet in den 90er Jahren hat sich TLF zu einer grossen Organisation diakonischer Arbeit in Pretoria entwickelt.

22 www.tlf.org.za (letzter Zugriff am 19.3.2017)

23 http://www.up.ac.za/en/centre-for-contextual-ministry/article/53984/staff (letzter Zugriff am 4.1.2017)
Beim Hauptsitz wurde ein Frauenhaus mit verschiedenen Räumen eingerichtet, darunter eine Kapelle. Hier können sich Frauen und ihre Kinder in Sicherheit wieder zurechtfinden. Sie finden hier professionelle Begleitung. Vor Jahren war in diesem Haus ein heruntergekommenes Bordell. Durch die aufsuchende Soziale Arbeit von TLF gelang es, die Frauen zum Ausstieg zu bewegen. Die Stadt übergab das Haus für 5 Mio Ran. Die ersten Mädchen zwischen 14-16 Jahren, die Prostituierte waren, waren auch die ersten jungen Frauen im Haus. Heute beherbergt das Haus Frauen und Kinder. 30 Kinder werden pro Tag zum Kinderhort gebracht. Angegliedert ist auch ein Altersheim mit 6-8 Zimmern. Hier ist der Grundgedanke von TLF wie auch von Diakonie festgebaut: Man geht nicht aus den Quartieren, sondern die Quartiere bestimmen die Soziale Arbeit. Es wird versucht, den Menschen in den Quartieren dazu zu verhelfen, dass sie besser und menschenwürdiger leben können. Dieses Frauenhaus ist ein gelungenes Beispiel für die Kontextualität der Diakonie auch im räumlichen und sozialräumlichen Sinne: Der Kontex bestimmt den Text des helfenden Handelns.

In einem Quartier, in dem auf kleinsten Raum 6000 Schwarze in Wellblech-Häusern ohne Wasser und ohne Elektrizität leben, hatte TLF zusammen mit Rotaryclubs auf einem Kirchgelände eine Villa abreissen lassen. Dort wurde ein Haus für 60 Kinder gebaut. Die Kirche, die nich mehr gebraucht wurde, wurde diakonisch umgenutzt und zum Gestaltungsraum für die Kinder umgebaut. Auf dem Boden waren Matten ausgelegt und ca. 30 Kindern lagen auf den Matten, schliefen oder bewegten sich auf die Anweisung der Kinderbetreuerin. Der hintere Teil der Kirche war abgetrennt und zu Räumen für den Kindergarten umfunktioniert worden. Das war eines der wenigen Beispiele einer diakonischen Nutzung einer Kirche, die nicht mehr für den Gottesdienst gebraucht wurde. 
Erfahrungsbericht

In einem Wohnheim für psychisch erkrankte Menschen habe ich mit der Leiterin Marlies Dauber über die Apartheid, Spiritualität und Diakonie und die Unterschiede zwischen Südafrika und Deutschland gesprochen:

- Rassismus in Deutschland ist anders als hier: In Deutschland wird der Rassismus durch die Gegend, Haarfarbe, Religion, den sozialen Status oder Gender definiert. Hier wird Rassismus durch die Farbe definiert. Sie muss den Weissen immer wieder sagen, dass auch sie das Haus putzen müssen.

- In Deutschland ist sie nicht nur aus der Kirche getreten, sondern auch nicht mit der Frömmigkeit in Beziehung gekommen, die sie hier antrifft. Spiritualität ist in den Alltag integriert in der Form, die ihr gut tut. Spiritualität ist eine Form von Deutung der Wirklichkeit, die den Wert dessen, was ist und nicht, was fehlt, gewichtet. In Deutschland ist an diese Stelle der Materialismus getreten.

- Im Unterschied zu Deutschland lautet der Grundsatz der Diakonie hier: Ich bin, weil Du bist. Dieser Grundsatz legt den Finger auf die Berufung (calling) der Arbeit. Die beiden Grundbegriffe sind Würde und Demut (dignity and bumble). Deshalb wird Helfen als Begleiten (journey) von Menschen interpretiert, in dem sich Geben und Nehmen die Waage halten.

Einmal im Jahr organisiert TLF zusammen mit dem Department of Christian Spirituality, Church History and Missiology der University of South Africa (UNISA) ein gemeinsames Essen. In diesem Jahr hatten der Departementschef der theologischen Fakultät der Universität, Derrick Mashau, Wilma de Beer (Stephan de Beer) und andere Pfarrer zu diesem Anlass eine Rede gehalten. Gegen Mitte der Veranstaltung waren ca. 100 Personen anwesend, davon vielleicht 60\% Obdachlose (homeless people). Derrick Mashau betonte den Traum Martin Luther Kings Jn., den die homeless zu träumen haben: «Hört nicht auf zu träumen! Die Bildung ist der Schlüssel zu allem. Niemand ist homeless oder alle sind homeless [...].» Wilma de Beer hielt eine flammende Rede für die Weiterentwicklung des Werkes.
Nach der Rede verteilte Wilma de Beer an ca. 10 homeless ein Zertifikat, das die Teilnahme an einem Kurs in kontextueller Bibelarbeit und Theologie belegt. Dies war ein neues Projekt und entsprach dem Bedürfnis nach Wissen und Spiritualität. Danach organisierte Wayne Akanani von TLF das Essen und betete selbstverständlich. Frauen hatten in der Küche des Kirchgemeindesaales der methodistischen Kirche das Essen vorbereitet. Derrick Mashau war in der Küche und half den Frauen beim Essen zubereiten. Der Professor war bei der Essensausgabe an die Obdachlosen am Ausschank.

Reflexion

Auch in Pretoria war eine grosse Kluft zwischen den Kirchen als Institutionen, den Kirchgemeinden und den diakonischen Werken zu beobachten. Die ev.-lutherische Kirche in Pretoria hat ein grosses diakonisches Werk, das jedoch in die Kirchgemeinden nicht integriert ist. Bei TLF geschah ein ähnlicher Prozess, der auch in der deutschen Diakoniegeschichte eingelagert ist. Die Initiative kam von den Kirchen, doch die Organisation trägt sich selbst. Der Gründer war als charismatische Figur notwendig. Aber die Organisation unterliegt einem Transformationsprozess weg vom Charisma hin zu Professionalität im sozialen Management und organisatorischen Strategieprozessen.

Die black culture hat das Feld von normativen Werte und Rituale übernommen. Diese Kultur war bis zur Lehre und Forschung an der Universität zu spüren: Man sprach frei, oberflächlich, ideologisch, wenig kritisch, unterschiedliche Positionen miteinander vergleichend. Es gab keine Horse-sol-Diakonie auch in dem Sinne, dass die Menschen mit ihren Kulturen und Ritualen das soziale Wirken prägen und ausgestalten.

Die Frage nach der interreligiösen Kontextualität der Diakonie hatte sich bis jetzt nicht gestellt. Das heisst: Das Klientel war multikulturell und multireligiös. Die Mitarbeitenden waren christlich geprägt von einer schwarzafrikanischen Frömmigkeit.

Der Staat hat sich aus der sozialen Verantwortung quasi verabschiedet: Er zahlt psychisch erkrankten Menschen eine soziale Rente von 
1300 Ran/Monat. TLF bot in einem Haus Plätze für solche Menschen an für 750 Ran inklusive alles.

Unbekannt ist der Markt der NGO's in Südafrika und Pretoria. Anscheinend ist er nicht so gross. TLF hat einen grossen Spielraum, sich zu entfalten. Die Vergrösserung von 11 auf 40 Mio Ran ist Ausdruck davon.

Barmherzigkeit und Gerechtigkeit küssten sich: Derrick Mashau zitierte Nelson Mandela, der in Pretoria gesagt hatte, dass das Teilen von Nahrung und Platz mit den homeless nicht ein Akt der Barmherzigkeit ist, sondern ein Akt der Gerechtigkeit. Diese grundlegende Verbindung hatte Benedict herausgearbeitet. Sie läuft einem neoliberalen Grundverständnis von Kirchesein zutiefst entgegen. Dennoch war sie unbestritten und wurde auch von der politischen Führung gesellschaftlich gesetzt. Hier haben wir in der Schweiz eine grössere Pluralität von politischen und kirchlichen Verantwortlichen. Liegt es daran, dass in Südafrika die Ungerechtigkeit zum Himmel stinkt und in der Schweiz ja alles unternommen wird, Armut unsichtbar zu machen? Je sichtbarer die Armut, desto politischer die Arbeit der Diakonie im Sinne von advocacy und Prophetie.

Das Grundthema fast jeder Biografie waren Geschichten von Gewalt und Missbrauch. Diakonie als helfendes Handeln hiess kontextuell hier: Heilung.

Weil das Geld an jeder Ecke fehlte, bedeutete Diakonie nur: Geld beschaffen.

Alltag und Sonntag waren die zwei Seiten derselben Medaille: Der Alltag wurde durchbetet, der Sonntag mit seinem Gottesdienst diakonisiert: Bis zu zwei Stunden gingen die Menschen zum Gottesdienst, d.h. der Gottesdienst war Sonntagsgestaltung zugleich.

Gottes Präsenz in der Welt war offensichtlich: Gott in der Stadt bedeutete nur Ausfluss des Bekenntnisses von Gottes Reich in dieser Welt und kann als kritische Kategorie gegen Apartheid und Rassismus politisch verstanden werden. Gottes Reich war aus der Geschichte heraus gelesen weiss bzw. das Reich des weissen Mannes.
Diakonie als theologische, kritische Kategorie kann nur als Aspekt einer öffentlichen Theologie verstanden werden, die feministisch und befreiungstheologisch das Inklusion-Exklusion-Paradigma durch Emanzipation, Versöhnung und Gemeinschaft zu überwinden sucht.

In einem Kontext von Diskriminierung und Rassismus kann Diakonie nur als prophetische Theologie betrieben werden. Deshalb wurden in den biblischen Arbeiten mehr prophetische Texte aus dem Alten Testament als Richtschnur zitiert als jesuanische Texte.

Das Paradigma der Apartheit hatte sich nicht verabschiedet, sondern kam in einer anderen, mutierten Gestalt als prägende Konstante in das öffentliche Bewusstsein. Die Black People hatten Angst, dass mit der Übernahme der Macht von den Weissen, die Apartheid neuen Aufschwung erhält. Die Schwarzen waren per se sehr stolz auf ihre Kultur. Die Weissen hatten nichts vorzuweisen, ausser das Grillen, denn sie kamen ja aus Europa und waren ja nur eklektisch ein Gemisch aus europäischen Versatzstücken europäischer Kultur: Ihre Sprache Afrikaans ist hör- und sichtbares Indiz dafür. Die Weissen kamen nicht vom Paradigma des Rassismus los: Beispielsweise musste der ganze Unterricht in Afrikaans abgehalten werden, dabei ist Zulu die Sprache der Mehrheit in Südafrika. Ausserdem durften Schwarze keine Führungspositionen einnehmen.

Da die Armut so drängend war, wurde in den Kirchen das Thema des Rassismus dadurch zurückversetzt. Indem sich vermehrt die Rassen mischen, wird die nächste Generation dies ändern. Diakonie als helfendes Handeln hatte demnach immer eine Priorisierung von sozialen Brennpunkten vorzunehmen und sie kontextuell einzuordnen.

\section{Liturgisch-therapeutische Wirkekraft}

Vom 17.-19. August 2016 fand in Pretoria der 11. Kongress «Consultation on Urban Ministry» zum Thema «we must rise up» mit 200 Teilnehmenden statt. Pastoren, Pastorinnen, sozial-diakonisch Tätige aus Südafrika, Afrika, USA und der Schweiz waren zu dieser Konferenz angereist. 
Zu Beginn setzten drei Personen ein Zeugnis, dass es wahr wurde: I have risen. Nachher beschrieben drei Pfarrer die Situation ihrer Arbeit in den Townships in Kapstadt und Kibera (Nairobi).

Besonders die Situation in Nairobi ist nicht vorstellbar: Auf neun Quadratmetern leben 5-köpfige Familien. Der Wohnraum ist ein grosses Problem. Die Arbeit mit den Pfarrern besteht vor allem aus Trainingsprogrammen, um die Bewohner und Mitglieder der Gemeinden zu ermächtigen. Hindernisse in der Arbeit sind: Theologisches Denken und Spiritualität sind nicht in den Alltag integriert. Die Gemeinden sind nicht genug tragend. Der Staat hat sich verabschiedet aus seiner Verantwortung Prozesse von Inklusion und Integration voranzutreiben. Der Glaube und die Tatsache, dass in den Townships bis zu 80\% Christen leben, gibt Kraft zum Weiterzumachen.

Die Frage nach den Frauen im Pfarramt wurde einhellig als eine fraglose Tatsache postuliert aufgrund des jesuanischen Verhaltens.

Erfahrungsbericht

In der Nähe von Soweto trafen sich Bewohner und Leiter des diakonischen Projektes für an HIV und Lepra erkrankte Kinder zum wöchentlichen Gottesdienst. ${ }^{24}$

Das Kinderheim mit 23 Plätzen etwas abseits war nur schwer erreichbar. Um 9.00 Uhr war der kleinere Raum mit ca. 20 Personen besetzt. Der Kantor sang schon. Die Jugendlichen und Kinder sassen auf der linken Seite, rechts waren die ersten Heimleiterinnen anwesend, ebenso ein alter Mann. Das Heim wurde 2001 von Trevor gegründet. Trevor ist in Soweto 1967 geboren. Er erlebte den Studentenaufstand am 16. Juni 1976 als 9-jähriger Knabe. Mit 17 Jahren wurde er Christ und arbeitete als ev. Pfarrer lange in Soweto selber. Mit der Gründung des Werkes, in dem HIV-Kinder und Lepra-Kinder aufgenommen werden,

24 Vgl. www.emthonjeni.org.za (letzter Zugriff am 19.3.2017). sowie Frauen in einer Werkstatt mit Kleidern, Arbeit und Würde bekommen, zog er an den Rand, zusammen mit seiner Frau. Seine Frau verstarb vor einem Jahr bei einem Unfall.

Der Gottesdienst am 22. August 2016 bekam durch die Musik seinen energetischen Fluss. Der Kantor verstand es, während den zwei Stunden improvisierend den Klangteppich zu legen zwischen leise und laut, rhythmisch und klanglich. Es war fantastisch, wie er die immer grösser werdende Gemeinde zum Klangkörper vereinigen konnte und so aus dem Raum einen Klangraum schaffte, der Resonanzraum für Theologie und Diakonie wurde. Die Lieder waren zum Teil überliefert, zum Teil auch frei improvisiert.

Die Liturgie pendelte zwischen Ordnung und chaotischem Freiraum. Leute kamen und gingen, am Schluss waren ca. 60 Personen anwesend. Ein musikalischer Klangteppich unterlegte das Sologebet vom Kantor und führte in eine Grundharmonie. Dann folgte ein freies Gebet von den Mitgliedern, dann die Hinführung zum gesungenen Unservater. Zur Begrüssung sprach Trevor zum ersten Mal. Er begrüsste die ausländischen Gäste, erwähnte seinen Besuch in Bern und auch Zwingli. Zwei Lieder folgten, die die letzten Gäste in den Raum zogen. Die Konzentration steigerte sich, die Atmosphäre verdichtete sich zu einem alle inkludierenden Gefühl: Nun geht es um uns und um mich.

Im Predigtteil (ca. 30 Minuten) sass eine Mitarbeiterin am Ende des Tisches. Sie schlug die Bibel auf und sagte, sie werde nun die Geschichte der Hure Rahab auslegen. Einzelne Mitglieder nahmen ebenfalls die Bibel in ihrer Sprache oder in Englisch und schlugen sie auf. Die Predigt war ein Gespräch und eine paradigmatische Unterweisung.

Gott braucht die Frauen, und zwar Rahab, als Prostituierte verstossen, als Lügnerin abgeschrieben, genauso wie die reiche Königstochter Esther, um sein Volk zu retten. Also, wir Frauen haben den Auftrag, dieses Verhalten der Frauen weiterzutragen in unseren Alltag. 
Mit Fragen zum Alltag von Gewalt, Gender und arm/reich, entsprach dies dem Bedürfnis der Gemeinde, ausser bei den vielen sehr kleinen Kindern, die jedoch auf den Plastikstühlen ruhig sitzen blieben.

Die Predigt wurde mit einer längeren Gesangslitanei abgeschlossen und führte in den emotional nun dichtesten Teil über. Dieser ca. $30 \mathrm{Mi}-$ nuten dauernde Teil hatte folgenden inneren Aufbau: Trevor erzählte vom Ereignis seiner Nichte, indem er die Nichte und eine Freundin nach vorne bat.

Die Nichte wurde am Freitag aus dem Taxi gerissen. Sie ist asthmakrank. Männer sprayten ihr in den Wagen. Sie bat, die Fenster zu öffnen, in dem Moment wurde sie gewaltsam aus dem Taxi gerissen. Die Männer liessen sie los, nachdem einige auf der Strasse zu ihr liefen. Die Gemeinde sei jetzt da, um in der Nachfolge von Jesus Christus ihr zu ermöglichen, ihre verletzte Seele heilen zu lassen.

Um diesen Raum für eine mögliche Heilung überhaupt zu schaffen, nahm Trevor sich als Beispiel und erzählte von seinem Besuch in Bern (Vineyard-Bewegung).

Im Gottesdienst kam eine Frau zu ihm, die von schwarzen Männern vergewaltigt worden war. Sie hielt ihn fest und bat ihn, ihr zu verzeihen. Das heisst, wir können stellvertretend für Täter Projektionsflächen werden, wo die Kraft Gottes zu heilen beginnt, indem auf dieser Fläche die Umkehrung von Gewalt in Versöhnung, von Verletzung in Heilung und von Hass in Liebe geschieht. Dass der Hass gestoppt wird sei deshalb so wichtig, weil der Hass sich wie Gift in eine Gemeinschaft verfliesst und so die Gemeinschaft, auch wenn sie Kirche genannt wird, vergiftet.

Daraufhin wendete Trevor sich zur Nichte und bat sie, sich von einigen Männern nun an den Schultern berühren zu lassen und von Wayne, einem Weissen, sich segnen zu lassen. Ebenfalls mutete er ihr zu, sich Gedanken zu machen und sich allenfalls auch dem Gedanken des Verzeihens einmal öffnen zu können. Nicht heute, nicht morgen, aber irgendwann einmal, um die Spirale von Gewalt zu stoppen. Er ging in die Gemeinde und forderte 5 Männer auf, sich um die Frau zu stellen, mittendrin die Freundin von ihr.
Der Kantor begann, mit seiner Musik einen therapeutischen Klangteppich zu legen. Wayne begann mit dem Gebet. Von einer WC-Rolle wurden durch den geschlossenen Kokon der Frau Tücher für die Tränen bereitgestellt. Fünf Minuten dauerte das Ritual des Gebets.

Trevor schritt ins Ritual ein, indem er sich aus dem Ritual des Gebetskreises und des Kreises derjenigen, die der Frau die Hände auflegten, herausnahm. Er öffnete den Kreis und reihte die Männer auf. Dann erklärte er, dass diese heilende Kraft Gottes nicht nur diese Frau erreicht, sondern alle Menschen, black and white, und auch das Land. Eine Form dieses Ausflusses war der politische Widerstand gegenüber Korruption und Gewalt. Nun begann er, die politischen Wahlen als Vorbereitung des letzten Schrittes dieses unglaublich dichten Moments und seine Interpretation zu interpretieren. Er verurteilte explizit das Verhalten von Zuma, drückte seine Hoffnung auf eine bessere Zukunft des Landes aus und bat die Gemeinde, den Blick nach aussen mit dem Blick nach innen zu verbinden. Stellvertretend für die draussen stehe die Gemeinde füreinander und miteinander ein. Dann bat er mich und andere nach vorne und bildete so ca. 6 Paare, die sich gegenseitig die Hände hielten und miteinander ins Gebet geführt wurden. Somit wurde die energetische Dichte, die sich im Kreis um das Gewaltopfer aufgebaut hatte, demokratisiert und kanalisiert, indem sie in die ganze Gemeinde und in die ganze Welt abgeleitet wurde. Das methodisch-theologische Prinzip war das der Stellvertretung, das diakonische Prinzip das von Mystik und Widerstand.

Ich hielt also die Hände der Frau, ich betete und bat Gott um Kraft für die Heilung der Wunden. Ich schwieg. Dann begann sie zu beten. Ihr Körper zitterte und gegen Schluss brachte sie über die Lippen: Gib mir die Kraft, Gott, auf deine Vergebung zu hoffen, wenn ich an mein Verhalten gegenüber den weissen Männern denke. Damit nahm diese Hausmutter eines Hauses, in dem vier Kinder auf kleinstem Raum wohnen, den Impuls von Trevor auf.

Als Schlussschritt führte Trevor in die Kollekte für die Gemeinde, in das letzte Lied und dankte der Predigerin für ihre erste Predigt. Das Lied öffnete wiederum die Türen, die immer offen standen und die Energie 
wurde mit der Musik förmlich nach draussen geweht. Während des Gesangs wurden schon die Stühle zusammengeräumt und die Umarmungen begannen.

\section{Reflexion}

Der Gottesdienst war ein offenes Kunstwerk folgender theologischdiakonischer Grundthemen:

Stellvertretung. Das Prinzip Stellvertretung wurde als hermeneutisches Prinzip in die Liturgie, in die Verteilung der Macht wie auch in die Befähigung der Gemeinde gelegt. Die Gemeinde wurde Stellvertreterin der Geschichte Gottes mit der Welt.

Mystik und Widerstand. Das mystische Einbinden des Glaubens in eine emotionale Intelligenz der Glaubenden gelang durch das Ineinander von Klang, Verteilung der Aufgaben und Leitung des Mystagogen, des Führers. Die Aufgabe des Pfarrers bestand in dieser Art darin, den mystischen und politisch widerständischen Teil in der Gemeinde und beim Einzelnen so miteinander zu verbinden, dass daraus der Strom des Heilenden und des Helfenden entsteht.

Diakonie als Heilung. Die Liturgie war Teil der Diakonie. In einer so kaum wahrgenommenen professionellen Selbstverständlichkeit verstand es Trevor, den diakonischen Aspekt liturgischen Feierns auf den heilenden Teil so hinzuführen, dass keine seelischen Übergriffe oder Blossstellungen geschahen. Dies konnte nur erreicht werden, indem sich die Gemeinde auch im Alltag kennt und das Leben miteinander teilt. Das heisst: Erst auf dem Boden des gegenseitigen Vertrauens konnte die Wirkung liturgischen Verhaltens dem Ursprung von Liturgie und Gottesdienst zugeführt werden. Als dies geschah, flossen Diakonie und Liturgie zum heilenden Geschehen an Betroffenen und stellvertretend für die Welt zusammen, ohne gleich zu werden.

Politische Diakonie und Theologie. Im Unterschied zu Heilungsgottesdiensten aus charismatischen Gemeinden war dieser Gottesdienst durchtränkt von der prophetischen Wirkung im Alltag. Der verbindende Ort war das Fürbittegebet. Die Haltung der Glaubenden entsprach der Stell- vertretung. Die Verkündigung war die Proklamation der Inanspruchnahme aller Frauen (und Männer) für diesen Dienst. Die Farbe der Menschen konnte eine Initialzündung dafür sein. Damit zeigte sich auf eine weitere Weise die Wirkung der Kontextualität von Diakonie, indem helfendes Handeln im liturgisch-therapeutischen Rahmen zum Klangteppich weiterer Ermöglichungen von helfendem Handeln wurde, konkret vor Ort und auf die Zeitachse verschoben in den nächsten Tagen und Wochen.

An diesem Morgen wurde sichtbar, was im westeuropäischen Kontext Bände füllt und mit dem Wort Eberhard Jüngels so zusammengefasst werden kann: «Diakonie als Tun des Guten spiegelt die Würde des Selbstverständlichen in der Welt.» ${ }^{25}$ Diese Würde des Selbstverständlichen zeigt sich in dem heilenden Beten für und mit Opfern von Gewalt als Ausdruck des Lebensbedrohlichem in dem Sinn, dass Opfer zu BotschafterInnen dieser Würde verwandelt werde.

\section{Streiflicht Nürnberg, 27.-28. September 2016 - identi- tätsstiftende Wirkkraft urbaner Diakonie}

Erfahrungsbericht

Nürnberg ist vom städtischen Kontext mit Zürich vergleichbar. Die Besuche bei der katholischen kirchlichen Arbeit der Offenen Kirche St $\mathrm{Klara}^{26}$, der Stadtmission ${ }^{27}$, der städtischen Sozialen Arbeit, dem Amt

25 Eberhard Jüngel, Das Opfer Jesu Christi als sacramentum et exemplum: Ders., Wertlose Wahrheit, München 1990, 261-282 (280).

26 http://st-klara-nuernberg.de/ (letzter Zugriff am 28.9.2016).

27 http://www.stadtmission-nuernberg.de (letzter Zugriff am 4.1.2017). 
für Existenzsicherung und Integration mit dessen Leiter Dieter Maly ${ }^{28}$, dem Lorenzladen mit dessen Leiter Thomas Zeitler ${ }^{29}$ sowie der Offenen Tür in der St. Jakobskirche mit deren Leiterin Barbara Hauck ${ }^{30}$ ergeben zusammen ein vielfältiges Bild diakonischer Arbeit im urbanen Kontext.

\section{Reflexion}

In der diakonisch-theologischen Reflexion lassen sich folgende Schwerpunktsetzungen aus den unterschiedlichen Projekten und Ansichten herausschälen, die kurz ausgeführt werden.

Konstitutive Verbindung von Spiritualität und Diakonie

Nimmt man Diakonie im städtischen Umfeld in den Blick, dann gewinnt man einen faszinierenden Blick auf die Diakonie, wie auch auf die Spiritualität.

Diakonie wird fast ausschliesslich als helfendes Handeln im Kontext von Kirche und Gesellschaft interpretiert, ein helfendes Handeln, das sich auf die jüdisch-christliche Tradition beruft, sich von dieser Tradition motivational beeinflussen lässt und sich so in andere Formen von helfendem, solidarischen Handeln von Staat und anderen NGO's einreiht. Damit zeichnet sich ansatzweise der stattfindende Paradigmenwechsel von einer exklusiv christologischen zu einer inklusiv schöpfungstheologischen Begründungs- und Deutungsstruktur im konkreten Vollzug von Diakonie in die Stadt ein. ${ }^{31}$

28 https://www.nuernberg.de/internet/sozialreferat/sozialamt.html (letzter Zugriff am 4.1.2017).

29 http://lorenzerladen.mws3.de/ (letzter Zugriff am 30.9.2016).

30 http://www.nuernberg-evangelisch.de/offene-tuer-cityseelsorge-an-st-jakob/ (letzter Zugriff am 30.9.2016).

31 Vgl. Rüegger / Sigrist (Anm. 4).
Diese so verstandene Diakonie wird jedoch immer in enger Verbindung mit Spiritualität theoretisch angedacht und praktisch ausgestaltet. Mit Spiritualität wird bei den Verantwortlichen meist die in der Volksfrömmigkeit angewandte Abgrenzung gegenüber der kirchlich verwalteten Frömmigkeit mit ihren althergebrachten Ritualen sprachfähig, indem Raum für alternative Rituale und Angebote interreligiöser Art geschaffen wird. Im Begriff Spiritualität liegt die Begeisterung für die Diffusität der geistgewirkten Erlebnisse und Erfahrungen eingelagert, die angesichts der Verletzlichkeit und Brüchigkeit der Stadtseele eine immer stärkere Evidenz gewinnt.

Diese hoch energetische und geisterfüllte Verbindung zwischen helfendem Handeln und spirituellem Erleben ist konstitutiv auf Raum ausgelegt und wird gleichsam als inkorporierte Pneumatologie in Gassen und Strassen, Kirchen und sakralen, öffentlichen Räumen eingezeichnet. Die Hilfe für Trauernde ausserhalb des Kirchenraumes und das öffentliche Ritual für Trauernde im Kirchenraum fliessen ineinander und lassen einen neuen Raum inmitten von Quartier und Stadt entstehen, in dem sich beides unvermischt und ungetrennt am gleichen Ort und gleichzeitig ereignet: helfendes Handeln, also Diakonie, und darstellender Glaube, also Spiritualität. Diese aus der christologischen Dogmatik entlehnte Konstruktion nimmt die prinzipielle Unterscheidung von darstellendem und wirkvollem Handeln des Glaubens von Friedrich Schleiermacher auf und entwickelt daraus diese für kirchliche Arbeit in der pluralen Gesellschaft grundlegende Dipolarität von Diakonie und Spiritualität, indem sie einen neuen Raumbegriff und einen neuen Missionsbegriff in den Stadtraum zeichnet.

\section{Sozialraum-Orientierung}

Unabhängig von den kirchlichen, diakonischen und staatlichen Trägern orientiert sich Diakonie als helfendes Handeln am Sozialraum. Damit werden für die Diakonie im urbanen Raum folgende Faktoren relevant:

- Gentrifizierung der Stadtquartiere,

- Transformationsprozesse in der Bevölkerung, 
- Ausschläge momentaner Krisenerfahrungen (Flüchtlingskrise),

- Differenzerfahrung in unterschiedlichen Stadtteilen und Quartieren,

- Ausrichtung auf konkret sich aufhaltendes oder wahnhaftes Klientel,

- Zusammenspiel von Räumen, Menschen und öffentlichen Plätzen.

Kirchgemeinden und Pfarreien setzen in dieser Sozialraum-Orientierung ihre Räume, ein Freiwilligennetz und Hoffnungsgeschichten von Einzelschicksalen als ihr symbolisches, ökonomisches, soziales und symbolisches Kapital ein. ${ }^{32}$ Für die Urbane Diakonie ist der Raum in seiner sozialen, politischen, ökonomischen und symbolischen Konturierung konstitutiv.

Reziprozität in der «Mission»

Durch die Pluralität hat eine schleichende und doch wahrnehmbare Verschiebung des Missionsbegriffes eingesetzt, die sich an folgender Beobachtung festmacht: Im alten Paradigma fühlten sich Christen in die Welt draussen gesandt, um Menschen für die Geschichte Jesu zu gewinnen und heimzuholen in ihre Gemeinschaft. Im neuen Paradigma verstehen sich Christen als Teil der pluralen Gemeinschaft, schaffen öffentliche Räume, sozusagen Allmenden missionarischen Potentials, indem sich Menschen unterschiedlichster Art begegnen und in dieser Begegnung miteinander ins Gespräch kommen. In dieser Struktur von Storytelling ereignet sich dann das Wort Gottes. So wird in neuer Art und Weise die Jesus-Geschichte in Pluralität und Säkularität mit ihren eigenen Verständnissen von Mission und Vision erzählt. Dieses Sprachereignis von Gesandt-Sein im öffentlichen Raum kann als Reziprozität von Mission

32 Vgl. zur Interpretation der Sozialtheorie Pierre Bourdieus auf kirchliche Arbeit: Sigrist (Anm. 15), 33-37.244-261. verstanden werden, indem Menschen wechselseitig zu Zeugenden und Hörenden werden: Zeugnis-Ablegende und Zeugnis-Empfangende. Oder mit den Worten Karl Barths zu reden: Christen als «Laufburschen» und «Laufmädchen» der Botschaft Jesu treffen in diesen öffentlichen Erzähl- und Schreibstuben auf andere Laufbuben und Laufmädchen und beide sitzen dann an einem Tisch ab und erzählen einander von ihren Geschichten. ${ }^{33}$ Damit scheint im so neu verstandenen Missionsbegriff eine erste Dimension vom «gemeinsam» in der Urbanen Diakonie auf.

Gemeinsam - Team-Play Urbaner Diakonie

In allen geführten Gesprächen wird deutlich, dass helfendes Handeln nicht mehr allein geht. Die Kirchen haben das Exklusivrecht für Humanität und Menschlichkeit verloren. Das Alleinstellungsmerkmal für «christliche Nächstenliebe» teilen sie mit säkularen und anderen religiösen Organisationen und Institutionen. Dieses «Gemeinsam» gilt nich nur für die Träger und für die leitenden Verantwortlichen der Organisationen und Institutionen. Der Ensemble-Gedanke ist nicht nur für Kirchgemeinden und Pfarreien in der Altstadt gefordert. Der TeamCharakter und der Ensemble-Gedanke sind vor allem für Urbane Diakonie fundamental und ziehen ihre Linien aus in das helfende Handeln selber. Die Reziprozität helfenden Handelns zeigt sich in der gemeinsamen Struktur von bezahlten, professionell ausgebildeten und freiwilligen Kräften. Diakonie arbeitet mit Freiwilligen und mit Betroffenen zusammen, nicht für Freiwillige und für Betroffene. Diese schon 1994 in der europäischen Bratislava-Erklärung festgehaltene prinzipielle Einsicht für die Diakonie wird in den nächsten Jahren für die Urbane Diakonie zur normativen und ethischen Grundkategorie, die sich dann im Füreinander konkretisiert.

33 Vgl. zum Bild Barths: Karl Barth, Das christliche Leben. Kirchliche Dogmatik IV $\backslash 4$, Fragmente aus dem Nachlass, Vorlesungen 1959-1961, Zürich 1976, 159. 
Füreinander - optionale Ausrichtung Urbaner Diakonie

Füreinander - dieser zentrale Begriff Urbaner Diakonie zeichnet eine Grundausrichtung helfenden Handelns aus, die die komplexe InklusionsExklusions-Struktur nicht im Sinne von Niklas Luhmann dipolar denkt sondern mit Martin Kronauer im Sinne einer gleichzeitigen Menschen ausschliessenden und einschliessenden Gesellschaft, die optional den Schwächsten immer wieder in den Blick nimmt. ${ }^{34}$ Helfen wird so als inkludierende Kraft verstanden:

«Als inkludierende Kraft grenzt sich das so verstandene helfende Handeln von einem Integrationsverständnis ab, das sozial, politisch oder kulturell marginalisierten Men schengruppen von «draussen` den Weg zur eigentlichen Gesellschaft «drinnen〉 eröffnen möchte. Die «draussen» gehören schon immer zu denen «drinnen»; ihre Begleitung und Unterstützung hat unter dem Gesichtspunkt ihrer Zugehörigkeit zu geschehen. Die inkludierende Kraft zieht beide, Helfende wie Hilfeempfänger, nicht in homogene, sondern in heterologogische Räumlichkeiten〉 beziehungsweise heterogene diakonische Räume, wo Platz für ein Miteinander unterschiedlichster Minderheiten geschaffen wird und mit «schrägem Blick» und «schräger Rede` die Vielfalt von Potenzialen und Fähigkeiten all dieser je auf ihre Weise «schrägen Vögel ins Spiel gebrach wird, um Diskriminierung, Marginalisierung, Benachteiligungen und Ausgrenzung abzubauen.» 35

34 Vgl. zu dieser soziophilosophischen Diskussion: Sigrist (Anm. 15), 112ff.

35 Sigrist (Anm. 14), 117. Ich gehe mit Ulf Liedke einig, wenn dieser sagt: «In Abgrenzung zum Mainstream des Integrationsverständnisses soll mit dem Inklusionsbegriff deutlich gemacht werden, dass sozial marginalisierte Menschengruppen immer schon zur Gesellschaft gehören und die Beseitigung von Diskriminierung deshalb als Grundlage der stets schon vorausgesetzten Zugehörigkeit erfolgen muss. Darübet hinaus geht das Inklusionsmodell nicht mehr von der Interaktion relativ homogene Personengruppen aus, sondern stellt die diversity, die Verschiedenheit, und Individualität der Menschen in den Mittelpunkt.» (Ulf Liedke, Theorie und Praxis der Inklusi-
Viele der sozialen Projekte werden in der Urbanen Diakonie mit diesem schrägen Blick (Bernhard Waldenfels) entworfen. Mehr noch: Der schräge Blick, der gegen die Normalität des Schauens bürstet, ist konstitutiv für das Innovationspotential in sozialen Transformationen.

\section{Quartierbezug Urbaner Diakonie: Ökumene und Interreligiosität}

Es gibt keine Horse-sol-Diakonie, sondern das Quartier ist Fundament und Raum Urbaner Diakonie. Dabei spielen Läden, Kaffees, Plätze, Museen und vieles mehr entscheidende Rollen für die Ausrichtung helfenden Handelns. Der Kirchenraum ist dabei ihr grösstes Potential und Kapital. Die diakonische Nutzung von Kirchenräumen schreitet voran (St. Jakob). Die Nutzungsverschiebungen in den Stadtkirchen richten das Angebot der Kirchgemeinden in neue Richtungen aus (St. Lorenz, St. Sebald). Die Transformationsprozesse und die Megatrends in der Gesellschaft schlagen unweigerlich auf die Nutzung der Kirchenräume aus Interreligiöse Nutzungsformen und ökumenische Zusammenarbeit sind keine Wahlmöglichkeiten mehr, sondern werden zur Norm kirchlichen Arbeitens in der Stadt. Theologisches Grundaxiom allen Arbeitens ist die jesuanische Frage, die neu an prägender Aktualität gewinnt: Wer ist ihm, dem Alleinstehenden, dem Flüchtling, der alleinerziehenden Mutter, etc. zum Nächsten geworden (Lukas 10,36)?

Die Frage nach der Identität im pluralen Kontext als Kernfrage Urbaner Diakonie Die Entwicklungen in den Quartieren werden zunehmend komplexer und pluraler. Dadurch stellt sich den kirchlichen Kreisen und den Menschen mit christlichen Glaubensüberzeugungen die Frage, wie die Identität neu aufgebaut werden kann angesichts des Weges zur Minderheit, zum Älterwerden und zu finanziellen Engpässen. Man kann die Urbane

on: Ders./Ralph Kunz (Hg.), Handbuch Inklusion in der Kirchgemeinde, Göttingen 2013, 11-29 (20). 
Diakonie als einen Aspekt christlicher Identitätssuche im säkularen Kontext verstehen, der in Anlehnung an die Entwicklung eines postsäkularen Begriffes von Religiosität, der sich auch in den Kirchenräumen zeigt, auf Entwicklungsströme zu einer postsäkularen Diakonie weist. Dabei of fenbaren sich der neue Bezug auf die jüdisch-christliche Tradition, der kritische Blick auf die Institution Kirche sowie die eigene Überforderung, christliche Existenz aufgrund der Herausforderungen von Menschen anderer Religionen aufzubauen. Man und frau verstehen sich als Pionierin, christlich begründete Oasen für Menschlichkeit und Spiritualität in der Stadt einzurichten. Die Stadt bekommt Züge eines Forschungslabors, um mit Experimentierfreude und Geistesgegenwart den ersten Schritt ins Offene zu wagen, ohne zu wissen, wo der zweite Schritt gesetzt werden kann. Doch diese Haltung zeugt vom Urvertrauen, das der christliche Glaube in den Werten von Glauben, Liebe und Hoffnung festmacht. Bei den Gehversuchen vergisst der Glaube nie, dass der Weg niemals am Kreuz vorbei ins neue Leben geht.

\section{Streiflicht Berlin, 13.-14. Oktober 2016 - urban- transformierende Wirkkraft Urbaner Diakonie}

Der Besuch der Tagung der Evangelischen Akademie zu Berlin «An den Rändern der Stadt. Urbane Peripherien» lenkte den Blick zum Schluss der Streiflichter auf die Urbanität selber.

Die Stadt ist nicht allein die City, die Innenstadt. Sie ist auch Vorstadt Peripherie, Speckgürtel, Stadtrandgebiet. Innerhalb dieser Bereiche finden sich erneut soziale, kulturelle und symbolische Zentren «zweiter Ordnung». Die moderne europäische Grossstadt stellt sich so als ein polyzentrisches Gebilde dar. Pointiert formuliert: Sie ist die Gesamtheit ihrer «urbanen Dörfen». Das lässt sich nicht nur in Berliner Kiezen beobachten, sondern ebenso in Wiener Bezirken und den Banlieues von Paris. Mit den Zentren pluralisieren sich auch die Peripherien. Welche Unterscheidungskraft liegt vor diesem Hintergrund noch im Begriff des «Stadtrandes»? Bei näherer Betrachtung erweist sich die Rede vom Rand als eigentümlich mehrdeutig. Einerseits ist die Peripherie weiterhin Teil der Stadt und zugleich von ihr unterschieden, ohne dass die Übergänge klar definiert wären. Andererseits schillert die Metapher des Randes zwischen einer geographischen und einer sozialen Bedeutung.

Ist der Stadtrand auch Rand des Geschehens oder heimliches Zentrum des Alltags? Wie bilden sich soziale Ränder der Gesellschaft in Siedlungsstrukturen ab? Wie lassen sich Aushandlungsprozesse zwischen sozialer und geographischer Randständigkeit in der Gentrifizierungsdebatte nachvollziehen? Welche Rolle spielen Kirche und Religion im Streben nach Integration der Peripherien und nach Gestaltung symbolischer Zentren in den Zwischenräumen der Stadt?

Zwei grundlegende Studien sind für die weitere Arbeit der Urbanen Diakonie wegleitend:

- Andreas Thiesen, Professor für Sozialarbeitswissenschaft in Leipzig, entfaltet in seiner Monografie die Frage: «Inwiefern führt die Diversifizierung urbaner Gesellschaften zu einer ambivalenten Konzeption sozialräumlicher Identität?» ${ }^{36}$ Die Frage nach der Identität ist demnach nicht nur ein kirchliches Thema, sondern als Ausdruck postmoderner säkularer Trends und Prozesse kann sie als Kristallisations-Zentrum individueller und kollektiver Sinnsuche verstanden werden. Dabei können die grundlegenden Postulate von Andreas Thiesen als Leitlinien für den städtischen Prozess von KirchGemeindePlus interpretiert werden: «Stadtentwicklung muss «das Lokale» neu denken und praktisch verhandeln - eine zentrale Problemstellung, findet Stadtentwicklung doch in erster Linie «vor Ort statt, im besten Fall im Aushandlungsprozess mit der Bevölkerung [...]. Reflexive Stadtentwicklung, so meine These, benötigt Mut zur künstlichen

36 Andreas Thiesen, Die transformative Stadt. Reflexion Stadtentwicklung jenseits von Raum und Identität, Bielefeld 2016, 19. 
Dummheit - womit ich die Fähigkeit zur Selbstreflexion und Selbstkritik thematisiere.. ${ }^{37}$ Beides, das Lokale neu denken und der Mut zur künstlichen Dummheit sind m.E. Grundbausteine für die Ausgestaltung von reformierter Kirche in der (urbanen) Gesellschaft.

- Hilfreich für diese Selbstreflexion und Selbstkritik scheint mir die Studie des Departements Architektur der ETH Zürich zu sein, die sich den Herausforderungen des Schweizerischen Nationalen Forschungsprogrammes «Neue urbane Qualität» (NFP 65) stellte und ihre Einsichten in einer Publikation veröffentlichten. ${ }^{38}$ Zum ersten Mal wird aus der Perspektive des Schweizerischen Kontextes Urbanisierung und Urbanität historisch, hermeneutisch und topografisch ausgeleuchtet und am Beispiel der «Metropolitanregion Zürich» konkretisiert. Die Forschenden postulieren: «Urbanität kann als Zustand umschrieben werden, indem unterschiedliche räumliche, soziale und ökonomische $\mathrm{Be}$ ziehungen stattfinden.»3 ${ }^{39}$ Sie definieren sechs Annäherungen: Zentralität, Diversität, Interaktion, Zugänglichkeit, Adaptierbarkeit und Aneignung. ${ }^{40}$ Dieses analytische Gerüst ist in sich konzis und so einleuchtend, dass es geradezu herausfordert, den kirchlichen Auftrag in der Urbanität damit neu zu überdenken.

Wie ist diese urban-transformierende Kraft aufgrund dieser Annäherungen zu beschreiben? Diese Frage ist eine der zentralen Herausforderungen in der Urbanen Diakonie, die in unterschiedlichen Publikationen und Veröffentlichungen zu unterschiedlichen Themen die Dozentur beschäftigen wird.

37 Thiesen (Anm. 36), 20.

38 Vgl. Kretz/ Kueng (Anm. 6).

39 Kretz / Kueng (Anm. 6), 43.

40 Vgl. zu diesen Annäherungen: Kretz / Kueng (Anm. 6), 43-73.
- Inwiefern können Kirchenräume mit deren Nutzungsverschiebungen in der City ihren Beitrag zum helfenden Handeln kirchlicher Institutionen erbringen?

- Inwiefern können Kirchgemeinden und Pfarreien mit ihrem Freiwilligennetz in den sozialen Brennpunkten wie bei den pflegenden Angehörigen von hochbetagten Eltern unterstützend wirken?

- Inwiefern sind Diakonie und Mission als Spannungsverhältnis zu klären und neu zu beschreiben?

Torsten Meireis, Ethiker an der theologischen Fakultät der Universität Berlin, hielt an der Tagung fest: «Es gibt ein moralisches Recht auf den Zugang zu einem normativ als öffentliches Gut verstandenen Raum, und zwar durch Teilhabe und Teilnahme als Gerechtigkeitsmotiv und als Anerkennung der jeweiligen Identität.» ${ }^{41}$ Mit Blick auf die Herausforderungen der Kirche postulierte er, dass die Verhinderung der Begegnung mit Fremden und die Tendenz zur Milieuverengung dem Glauben widersprechen. Räume werden für darstellendes und wirksames Handeln gebraucht: Sie sind kollektiv, religiös, affizierte Praxis. Ein wirksames Gerechtigkeitshandeln richtet sich auf günstigen Wohnraum, Bildungs- und Erholungsräume, öffentliche Plätze und virtuelle Räume für politische Arenen. Es geht um diese Balancierung zwischen darstellendem und wirksamem Handeln. Dies ist deutlich zu machen nicht wegen Missionierung oder Dankbarkeit. Sondern in einer pluralen Gesellschaft ist entscheidend, den Hintergrund aufzuzeigen, aus welcher Geschichte und warum man was tut - das himmlische Jerusalem mit den offenen Toren: erzählt mal, warum ihr das macht?

41 Thorsten Meireis, «Recht auf Zugang? Sozial-ethische Überlegungen zur Gentrifizierung des öffentlichen Raumes», nicht schriftlich vorliegender Vortrag an der Tagung «An den Rändern der Stadt. Urbane Peripherien» in Berlin, 13.-14. Oktober 2016. 
In diesen zusammenfassenden Punkten ist ein ganzes Programm Urbaner Diakonie enthalten, das es in den nächsten Jahren in dieser der Diakoniewissenschaft eigenen Kongruierung von Theorie und Praxis interdisziplinär zu entwickeln gilt.

\section{Autor:}

Christoph Sigrist, PD Dr., Dozent für Diakoniewissenschaft an der Universität Bern und Pfarrer am Grossmünster in Zürich

\section{Anhang zu «Streiflichter Urbaner Diakonie»}

\section{Erfahrungsbericht von Luisa ${ }^{42}$}

«My story is quite a long one but in summary it began with me leaving home, ending up on the street, being physically abused, being in abusive relationships, multiple rapes and having to live with the consequences. I was infected with the HIV Virus and I live with major depression and anxiety disorder.

But this is my past; I do not see myself as a victim but as a victor.

In line with today's theme \#we must rise - I have risen.

I believe that my past mistakes were not meant to define me but to guide me. When I look in the mirror I do not see a victim of rape and abuse, I don't see a homeless person, I don't see a person who is infected with HIV or one who is living with a mental illness. No ways I see Louise. The Louise who has risen to what God created me to be. My Father in heaven would never put me through situations that I could not handle and not use for His purpose in the kingdom. No way!! That is why I believe I am here today

I have a number of stories relating to how I was sexually abused. I lost my virginity through sexual violation because I was afraid that the man I was with would throw me out into the street if I didn't consent. I think from that day onwards I was damaged. I had grown up with the expectation that I would find a partner who I loved with whom I would share my first sexual experience. So this was destroyed. I never felt the same way about myself where sex and intimacy were concerned. I stayed with an abusive man when I was in the East Rand, he didn't think he was being abusive because he thought he was taking care of me by putting a

42 Diese Lebensgeschichte ist zu beziehen bei: www.christophsigrist.ch. 
roof over my head and in return I had to let him have his way with me. I tried to leave him but ended up being raped by a gang of street kids ranging from 8 to 14years. I think this was the most traumatic experience I've ever had. I was much older than they were yet they overpowered me and took turns raping me. After this I ended up leaving eventually and went to Randburg where I also was in another relationship that I didn't want to be in but had to be just so that I would have a place to sleep.

I realize now that I never really knew how many times I had been sexually violated until I started talking to Psychologists and therapists because I had blocked out the memories. I had disconnected and had stopped feeling anything anymore and then I would drink alcohol to try and forget.

Shortly after this incident I left and came to Pretoria. With all these incidents I had come to a point where I didn't have any problem with giving my body away in exchange for something because this is what I'd learnt. So if I needed, shelter, food, alcohol I would use my body in exchange. At least it was with my consent and I'd already lost my dignity. I thought I was trying to survive, make the best of things and basically just stay alive and I thought I had no other options but I was actually dying inside.

My story started changing when I came across The Potter's House, at a time when I think God knew that I was ready for a change in my life. When I arrived in Pretoria I did not know anyone. I found myself on the street with nowhere to go. I had little money on me which quickly ran out and I found myself in the hands of people who took advantage of me, mainly men.

But before the week was over I was rescued and I met someone who took me to The Potter's house. I was grateful from the day I walked into the shelter.

After not having bathed or showered for days I still remember the relief I felt. I then had my first proper meal in days. That night when I slept I could not believe that I was sleeping in my own bed and I didn't have to pay for it with my body or money.
Jeremiah 18 (New King James Version NKJV)

The Potter and the Clay

The word which came to Jeremiah from the Lord, saying: 2 Arise and go down to the pot ter's house, and there I will cause you to hear My words. 3 Then I went down to the potter's house, and there he was, making something at the wheel. 4 And the vessel that he made of clay was marred in the hand of the potter; so he made it again into another vessel, as it seemed good to the potter to make.

There my journey at The Potter's House began and a new life with God. I was blessed with people who were genuinely concerned about me. God kept on giving me people until he had blessed me with an entire community. The people at the Potter's House believed in me and saw my true potential. The Potter's House had a holistic program that met all my needs. I got the Psychological help that I needed and a Social worker helped to reunite me with my family. Whilst I was there I began to get sick and found out that I was infected with HIV but fortunately I was in the right place and I got the help that was necessary. Eventually I became empowered through The Potter's House in many aspects. I also got support academically and I went to Hotel School where I discovered skills and talents that I'd buried away.

I even got my first real job as a House Mother at The Potter's House. A position of great responsibility. I had become a leader and inspiration to others. I then worked at an orphanage where I was a mother to five children who had lost their families due to HIV and AIDS. I had leave because I still had a lot of unresolved personal issues. Mainly because what had happened to me had really affected my whole perception of the world and I didn't really know how to live in it. It hasn't been easy to adjust and adapt, because I lived in a world where I felt I didn't belong and worst of all I didn't love myself.

But I have learned that life is not about what happens to me; it's what I do about it. This is how I rose. I have learned that I shouldn't compare myself to the best others can do but be the best me I can be. I have learnt that it isn't always enough to be forgiven by others, sometimes you 
have to learn to forgive yourself. As a result my life has begun to change. I have learned that I can keep going long after I think I can. I believe that I'm here today for a reason. I am here so that someone can identify or relate with me and be encouraged to rise. If I can inspire just one person to rise beginning today I will have made a difference. I believe that everything happens for a reason and this is part of God's purpose for me and through Him I have the strength and courage to accomplish this.

Life was not easy. I got knocked around by the trials I faced. But I realize that even the most successful people struggle or have struggled. The key is for an outcome that benefits me. What matters most, what carried me through the rough times, is what I was able to acquire, learn, build upon and harness over the course of many years. This was: a quiet sense of inner determination. I was determined somehow to survive.

I have now turned my adversity to my advantage. By helping others, sharing my experiences, strength and hope, I help myself to heal.

I didn't survive all that I went through just to throw everything away. I believe I can and will make it. I am somebody and I'm worth it.

Helen Keller wrote: «Character cannot be developed in ease and quiet, only through experience of trial and suffering can the soul be strengthened, ambition inspired and success achieved.)

I now work for the Department of Health as a Housekeeping Supervisor. From my journey with the Potter's House I became a fully integrated resident of the city. My relationship with the Potter's House is still very strong. I will never forget everything they did for me. I am now at a point in my life where I am ready to give back and help others because I really believe that my experiences can help others. I'm planning to study Social Work so I can work with NGO's like the Potter's House or even the Potter's House itself or even open my own Potter's House. I used to in the problem, was a part of the problem, now I need to and want to be in the solution.

There is a song that says God says: «Come away with me, come away with me, it's not too late; it's not too late for you. I have a plan for you
[...] It's going to be awesome [...] It's going to be great. It's going to be full of me. God's plan for me is full of opportunities interesting possibilities, exciting challenges and a life that will last forever.»» 